\title{
Finite Element Modeling of Branched Ruptures Including Off-Fault Plasticity
}

(Submitted 8 May'11 to the Bulletin of the Seismological Society of America, re-submitted with revisions 9 Oct '11.) (Accepted for publication 18 Oct '11.)

Nora DeDontney

Dept. of Earth and Planetary Sciences Harvard University

20 Oxford St., Cambridge MA, 02138, USA

(ndedontn@post.harvard.edu)
Publication citation:

Bulletin of the Seismological Society of America, Vol. 102, No. 2, pp. 541-562, April 2012, doi: $10.1785 / 0120110134$

James R. Rice

Dept. of Earth and Planetary Sciences and School of Engineering and Applied Sciences

Harvard University

29 Oxford St., Cambridge MA, 02138, USA

Renata Dmowska

School of Engineering and Applied Sciences

Harvard University

29 Oxford St., Cambridge MA, 02138, USA 


\section{Abstract}

Fault intersections are a geometric complexity that frequently occurs in nature. Here we focus on earthquake rupture behavior when a continuous, planar main fault has a second fault branching off of it. We use the finite element method to examine which faults are activated and how the surrounding material responds for both elastic and elastic-plastic off-fault descriptions. Compared to an elastic model, a non-cohesive, elastic-plastic material, intended to account for zones of damaged rock bordering maturely slipped faults, will inhibit rupture on compressional side branches and promote rupture of extensional side branches. Activation of extensional side branches can be delayed and is triggered by continued rupture propagation on the main fault. We examine the deformation near the branching junction and find that fault opening is common for elastic materials, especially for compressional side branches. An elastic-plastic material is more realistic since elevated stresses around the propagating rupture tip and at the branching junction should bring the surrounding material to failure. With an elastic-plastic material model, fault opening is inhibited for a range of realistic material parameters. For large cohesive strengths opening can occur, but with material softening, a real feature of plastically deforming rocks, opening can be prevented. We also discuss algorithmic artifacts that may arise due to the presence of such a triple junction. When opening does not occur, the behavior at the triple junction is simplified and standard contact routines in finite element programs are able to properly represent the physical situation. 


\section{Introduction}

Earthquake ruptures are rarely confined to a single planar fault, but rather propagate through various geometric complexities including step-overs, bends and branches. While the likelihood of rupture propagation through all of these features is important for understanding the interactions between faults, we confine our discussion here to fault intersections where there is a through-going, straight, main fault and a second fault, the branch, that intersects the main fault.

The dynamics of earthquake rupture through branched geometries has been studied (e.g., Aochi et al., 2000; Kame et al., 2003; Bhat et al., 2004; Duan and Oglesby, 2007), but the physical processes that take place at the branching junction have not been thoroughly examined. The presence of the triple junction introduces physical and algorithmic complexities that require attention. These include the issue of fault opening in the vicinity of the triple junction and how finite element procedures implement the interactions at the fault intersection. The material model (elastic versus elastic-plastic) alters the physical process and the resulting deformation near the junction may or may not be adequately described by standard finite element contact procedures.

\subsection{Background on Fault Branch Geometries and Dynamic Rup- ture Modeling}

Ando et al. (2009) use a California based study to show that examples of branched fault geometries are numerous at a range of length scales. They find that true Yshape geometries are rare and the dominant geometry is of one planar fault through the junction and a branch fault intersecting this at an angle of $17^{\circ}$. The dominant geometry is the same for all length scales investigated. The branches are equally distributed on both sides of the fault, so in a strike-slip setting, branches that exist in the compressional side of a propagating rupture are equally as common as those in the extensional side (see figure $1 \mathrm{~b}$ for a definition of compressional and extensional side branches). The equal distribution on both sides would presumably not be retained in the thrust fault setting, where most branches exist in the hanging wall, or the compressional side of the fault.

Additional recent work has considered how branch faults form. Scholz et al. (2010) explain the occurrence of fault branches as a result of a rotation of the principal stresses over time. This leads to branch formation when the main fault rotates out of 
optimal orientation and the stress state is able to cause failure on a new fault plane. Ando and Yamashita (2007) model the nucleation and propagation of shear branches extending from a predefined main fault. They see that one branch can become dominant over other branches and this may be the start of a macroscopic branch like we observe in nature.

To investigate the likelihood of these branch faults rupturing during an earthquake on the main fault, Poliakov et al. (2002) considered the dynamic stress field around a propagating mode II crack. Using a slip-weakening friction law on a preexisting fault in an elastic medium, they found regions where the stress field exceeded the Mohr-Coulomb failure criterion and plastic deformation should occur. The location of this region was shown to depend on rupture velocity and the orientation of the most compressive stress. Branch faults located in these highly stressed areas could potentially rupture during a dynamic event.

Models of rupture propagation through branch geometries assume the location of the branch a priori, and have primarily focused on strike-slip events. Bhat et al. (2004) studied the 2002 Denali event, Fukuyama and Mikumo (2006) studied the 1891 Nobi event, and Oglesby et al. (2003) examined the 1999 Hector Mine event. The surface rupture of the 1992 Landers earthquake activated multiple fault branches and illustrates the complexities of a rupture path (Sowers et al., 1994). Fliss et al. (2005) studied backwards branching in this event and Bhat et al. (2007) considered the interaction between the main fault and finite length branches. In addition to these strike-slip studies, Kame et al. (2003) addressed the presence of branched faults in thrusting regime and Templeton et al. (2010) investigated branch activation during normal faulting.

Kame et al. (2003) identified the dependence of the rupture path selection on the stress state (specifically the angle, $\Psi$, that the most compressive principal stress makes with the main fault), the branch angle $\delta$ (the angle between the main fault and the branch), and the velocity of the rupture, $V_{r}$, on the main fault at the branching junction. Both $\Psi$ and $V_{r}$ alter the stress field around the propagating rupture tip

(Poliakov et al., 2002) and depending on $\delta$, this may or may not lead to rupture on the branch fault.

\subsection{Objectives}

We seek to address both physical and algorithmic issues that arise due to the presence of the triple junction. Physically, we focus on the material description and how this 
alters the deformation around the triple junction as well as the rupture behavior at the junction. Algorithmically, we address the finite element (FE) implementation at the fault intersection. We discuss FE contact procedures and how artifacts of these affect the branch activation results, a problem when opening occurs at the junction.

Physically, McKenzie and Morgan (1969) pointed out that fault triple junctions are unstable; the geometry is not maintained when slip occurs and an opening at the intersection is necessitated. Andrews (1989) also closely examined the mechanics of fault interactions at fault bends and branches, and found that opening could occur at a triple junction. He determined that after multiple earthquake cycles, the resulting void would become a barrier to rupture and a fresh fracture would need to be generated in the area of the junction. Andrews (1989) also determined that the stress concentration due to slip at a fault bend would require slip on an associated spur fault.

We consider both elastic and elastic-plastic material descriptions to determine when opening is predicted in the vicinity of the triple junction. Opening should only occur, for the geometries studied here, for elastic material models and elastic-plastic models with large cohesive strengths. We also examine how the material description changes the branch activation. The stress field around an elastic rupture tip is very different from that of an elastic-plastic rupture tip. We examine these differences and discuss how the stress field affects the rupture path selection.

Proper assumptions and computational model implementation are key for an accurate interpretation of the likelihood of multi-segment ruptures. Branch activation has been examined using a variety of numerical approaches including the Boundary Integral Equation (BIE) method, Finite Element (FE) and Finite Difference (FD) routines. Depending on the numerical implementation, a choice may have to be made at the branching junction. Here we use the FE method, and while the FE procedure inherently has no problem handling a triple junction geometry, FE contact procedures can require specifications at the junction. These algorithmic choices can include how the faults are able to slide and if opening can occur. We discuss these choices and what is valid in the case of no fault opening. In certain cases, like when fault opening does occur, we show how these specifications can affect branch activation.

\section{Model Setup}

We used the explicit dynamic finite element package ABAQUS/Explicit to investigate branch activation in a $2 \mathrm{D}$ plane strain model. The faults are predefined and imbed- 
ded in an otherwise homogeneous full space (figure 1). The model is surrounded by absorbing elements, which minimize reflections from the boundaries, and here we do not take into account the presence of a free surface or depth dependent stress states.

The stress states used in this analysis result in right lateral rupture and stresses are positive in tension. They are defined by an initial main fault normal stress, $\sigma_{y y}^{0}$, fault parallel stress, $\sigma_{x x}^{0}$, shear stress, $\tau_{x y}^{0}$, and plane perpendicular stress, $\sigma_{z z}^{0}$, which is only relevant for elastic-plastic models. The stress state can be characterized by a prestress angle, $\Psi$, which is the angle between the most compressive principal stress and the main fault, and an $S$ ratio on the main fault. The $S$ ratio is defined as $S=$ $\left(\tau_{p}-\tau_{x y}^{0}\right) /\left(\tau_{x y}^{0}-\tau_{r}\right)$ where $\tau_{p}$ and $\tau_{r}$ are the peak and residual shear stresses respectively, based on the fault friction, $f$, and $\sigma_{y y}^{0}$. For sufficiently low seismic $S$ ratios, $S<1.77$, the rupture can transition from sub-Rayleigh to supershear rupture velocities by the formation of a daughter crack ahead of the main rupture (Andrews, 1976). While stress states are used in which supershear rupture is possible, here we only examine rupture behavior for cases of subsonic rupture velocities at the branching junction.

We consider two values for $\Psi$ and examine both compressional and extensional side branches, $-30^{\circ} \leq \delta \leq 30^{\circ}$ (figure 1b). For right lateral configurations, and a rupture propagating to the right, compressional side branches are in the top half of the model $(\delta>0)$, and extensional side branches are in the lower half of the model $(\delta<0)$. Previous work by Kame et al. (2003) has shown that compressional side branches can be activated for low values of $\Psi$, and extensional side branches can be activated for high values of $\Psi$. We use $\Psi=13^{\circ}$ and $\Psi=47^{\circ}$ to examine compressional and extensional side branches, respectively. These angles are chosen for the range in behaviors exhibited for the stress states we consider (we examine stress states with $1.0 \leq S \leq 3.0$, although not all results are shown here). For example, with $\Psi=55^{\circ}$, all extensional side branches in an elastic model are activated, but for $\Psi=40^{\circ}$, almost no extensional side branches will be activated (for the parameters considered here). Therefore we use $\Psi=47^{\circ}$ because it is in the transitional regime and activation is sensitive to parameter choices.

\subsection{Mesh Geometry and Element Definitions}

The FE model is composed of 4-noded linear rectangular elements and 3-noded linear triangular elements (types CPE4R and CPE3 in ABAQUS). The domain boundaries are 4-noded absorbing elements (Lysmer and Kuhlemeyer, 1969) (type CINPE4 in ABAQUS), which are perfectly effective when the incident wave front is parallel to 
the absorbing elements. Along the fault, the element size, $\Delta x$, is chosen such that the slip-weakening zone size is well resolved, $\Delta x=R_{0} / 40$, where $R_{0}$ is the nominal static slip-weakening zone size (figure 2). The slip-weakening zone size is the important length scale in the problem, and the element size is chosen to be certain that this length scale is well resolved. Even with contraction of the slip-weakening zone as the rupture accelerates, there are still $\sim 10$ elements in the zone, indicating that increased mesh refinement is not necessary. To verify this, for some test cases we implement an element size of $\Delta x=R_{0} / 60$ and find no difference in branch activation results.

Some previous models have used a uniform element size throughout the model domain, but this is computationally inefficient so we use an non-uniform element size, increasing with distance from the fault (figure 1). A uniform element size with $\Delta x=R_{0} / 40$ exists within a predetermined range from the fault and extends to 5 $16 R_{0}$ depending on the FE model run and if off-fault plasticity is included. This is bounded by six layers of progressively increasing element size, composed of triangles and squares, which are followed by more layers of the largest elements. By testing multiple geometries and stress states, we find that the branch activation results do not differ for a mesh with a uniform element size throughout the model domain and the coarsening mesh used here.

The mesh is predominantly composed of elements with a $\sim 1: 1$ aspect ratio to minimize the number of elements required to fill the model domain. The precise geometry of the elements around the branch is dependent on the branch angle, but all meshes are qualitatively similar to that shown in figure 1.

Elements with a large aspect ratio are still required in the corner of the branching junction, and since it is the smallest element that determines the time step, we increase the density of the few small elements so that the time it takes the $\mathrm{P}$ wave to travel across these elements is the same as for the regular fine resolution elements. This decreases the run time and we do not see a change in model results.

We employ a contact procedure on the faults that effectively reduces to a split-node procedure (see Templeton and Rice (2008), appendix B, for a complete description). Within that implementation in the FE package, ABAQUS, fault surfaces participate in contact interactions (there is an interaction that defines the main fault and an interaction that defines the branch), and a node cannot belong to two surfaces if each surface is involved in different contact interactions. This is not a feature common to all $\mathrm{FE}$ implementations, but rather an algorithmic issue due to the programming of the contact procedure. A decision must then be made for each of the nodes at the branching junction. Specifically, in figure $3 \mathrm{~b}$, do nodes 1 and 2 participate in the main 
fault or the branch fault contact interaction?

Our default model setup is that which constrains slip to occur only on the main fault at the branching junction. This is accomplished by defining the elements in such a way that the two sides of the branch fault merge at the junction, i.e., nodes 1 and 2 are actually the same node, as shown in figure $3 \mathrm{~d}$. This model definition prescribes that slip on the branch fault goes to zero as the branching junction is approached. This must be true if there is no opening of the main fault, because as shown in figure $3 \mathrm{c}$, branch slip at the junction leads to opening of the main fault. This default mesh definition implies no artificial algorithmic constraint if there is no fault opening, but there is no physical basis to make this choice in the event of fault opening.

\subsection{Elastic and Elastic-Plastic Materials}

For the model cases studied here, we use a homogenous, isotropic elastic or elasticplastic material (see table 1 for a complete list of parameters). We specify a representative density, $\rho$, of $2700 \mathrm{~kg} / \mathrm{m}^{3}$, as well as a Poisson's ratio of $\nu=0.25$, and a compressional wave speed, $C_{p}=5200 \mathrm{~m} / \mathrm{s}$. This results in a Young's modulus, $E=61 \mathrm{GPa}$, shear modulus, $G=24 \mathrm{GPa}$, and shear wave speed, $C_{s}=3002 \mathrm{~m} / \mathrm{s}$.

To simulate an elastic-plastic material, we use the pressure dependent DruckerPrager yield criterion, given by

$$
\bar{\tau}-\mu p=b
$$

where $b$ is the cohesion, $p$ is the pressure, $p=-\sigma_{k k} / 3$, for a stress tensor, $\sigma, \bar{\tau}=$ $\sqrt{(1 / 2) s_{i j} s_{i j}}$ is the second invariant of the deviatoric stress $s_{i j}$, with $s_{i j}=\sigma_{i j}+\delta_{i j} p$, and $\mu$ is the slope of the yield surface in the $\bar{\tau}$-p space (figure 4). For the initial stress states used here, in which $\sigma_{z z}^{0}=\left(\sigma_{x x}^{0}+\sigma_{y y}^{0}\right) / 2$, the Drucker-Prager yield criterion coincides exactly with the Mohr-Coulomb (M-C) criterion

$$
\max \left[\tau-\sigma_{n} \tan \phi\right]=c
$$

where $c$ is the cohesion, $\phi$ is the angle of internal friction, $\tau$ and $\sigma_{n}$ are the shear and normal tractions on any plane ( $\sigma_{n}$, positive in compression, is defined by $\sigma_{n}=-n_{i} \sigma_{i j} n_{j}$ for surface normal $\mathbf{n})$. For these stress states, the two yield criteria are related by $b=c \cos \phi$ and $\mu=\sin \phi$, although this exact agreement is lost as stresses vary during rupture.

When off-fault plastic deformation is included, we report contours of accumulated 
plastic strain, $\gamma_{e q}^{p l}$, where

$$
\gamma_{e q}^{p l}=\int_{0}^{t} \frac{d \gamma^{p l}}{d t^{\prime}} d t^{\prime}
$$

and $d \gamma^{p l}=\sqrt{2 d e_{i j}^{p l} d e_{i j}^{p l}}$, and $d e_{i j}^{p l}=d \epsilon_{i j}^{p l}-\delta_{i j} d \epsilon_{k k} / 3$ for a strain tensor, $\boldsymbol{\epsilon}$.

When investigating fault opening at the junction, we consider a range in cohesive strengths. If a representative normal stress at seismogenic depth is $\left|\sigma_{y y}^{0}\right|=100 \mathrm{MPa}$, the cohesive strengths we consider have the range, $60 \mathrm{~Pa} \leq b \leq 30 \mathrm{MPa}$. This is the same as a M-C cohesive strength of $70 \mathrm{~Pa} \leq c \leq 35 \mathrm{MPa}$, and $c=35 \mathrm{MPa}$ is a high cohesive strength for a range of rock types (Carmichael, 1982). If we assume that the fault is well developed and multiple ruptures have passed through, the fault will be surrounded by highly granulated rock. When that rock can be regarded as effectively cohesionless, $b=60 \mathrm{~Pa}$ is representative cohesion value (given its smallness compared to stress changes of order one to several MPa during rupture). If, however, significant cementation occurs during the interseismic time, cohesive strength can be partially or fully regained and higher values for cohesion are relevant.

We also allow some amount of dilatancy, and sometimes hardening, to occur during the plastic deformation. We define the dilatancy factor, $\beta$, as the ratio of an increment of volumetric plastic strain, $d \epsilon_{k k}^{p l}$, to an increment of shear plastic strain, $d \gamma^{p l}$

$$
\beta=\frac{d \epsilon_{k k}^{p l}}{d \gamma^{p l}}
$$

where $d \gamma^{p l}$ is defined in eq. (3). The range for $\beta$ is $0 \leq \beta \leq \mu$, where $\beta=0$ is nondilative and $\beta=\mu$ is associated flow. We investigate dilatancy values of $\beta=0.256$ and 0.389. Material hardening $(h>0)$ or softening $(h<0)$ is defined as

$$
h=\frac{d b}{d \gamma^{p l}}
$$

and it describes how the yield surface shifts as plastic shear deformation occurs (figure 4). If $h>0$, the yield surface shifts up in $\bar{\tau}-p$ space, and the material gains cohesive strength, i.e., it hardens. If $h<0$, the yield surface shifts down, and the material can lose all cohesive strength, i.e., it softens.

\subsection{Fault Constitutive Behavior}

This study differs from many earlier works of fault rupture propagation by the implementation of a regularized form of slip-weakening. The linear slip-weakening for- 
mulation proposed by $I d a$ (1972) and the general slip-weakening form proposed by Palmer and Rice (1973), depend only on slip, but the regularized form introduces a time scale that builds on the slip-weakening formulation as described below.

\subsubsection{Linear Slip-Weakening}

We define $f_{s w}$ as the slip-weakening coefficient of friction, which depends on the amount of slip on the surface, $s=s(x, t)$, where $x$ is position and $t$ is time. The strength of the surface, $\tau$, depends on the coefficient of friction $f$ and the normal stress, $\sigma_{n}=-n_{i} \sigma_{i j} n_{j}$, such that

$$
\tau=f \sigma_{n}
$$

where $f=f_{s w}$ except in the region of rupture nucleation (discussed in section 3.4).

We adopt the linear slip-weakening formulation (figure $2 \mathrm{~b}$ ), in which the coefficient of friction, $f_{s w}$, decays linearly from a peak static value, $f_{s}$, to a residual dynamic value, $f_{d}$, over a characteristic amount of slip, $D_{c}$, according to the law:

$$
f_{s w}(s)= \begin{cases}f_{s}-\left(f_{s}-f_{d}\right) \frac{s}{D_{c}}, & s<D_{c} \\ f_{d}, & s \geq D_{c}\end{cases}
$$

Using this law, the strength of the fault linearly decays from a peak value of $\tau_{p}=f_{s} \sigma_{n}$, to a residual value, $\tau_{r}=f_{d} \sigma_{n}$. In the event of fault opening, $f_{s w}$ does not continue to evolve. This is because there is no accumulation of "slip" in its sense as the variable on which the friction coefficient $f_{s w}$ depends, although the surfaces can continue to displace tangentially relative to one another. If contact across the fault is later reestablished (not a phenomena observed in most simulations), the evolution of friction resumes from its prior, potentially weakened value.

This formulation does not take important dynamic weakening effects into account, but has successfully been used to model earthquake rupture in single fault models (e.g., Duan and Oglesby, 2005), fault step models (e.g. Harris and Day, 1999) and branched geometries (e.g., Aochi et al., 2000; Kame et al., 2003; Templeton et al., 2009).

A length scale that arises is the slip-weakening zone size, $R$, the distance between the crack tip and the area of the fault that has undergone complete weakening (figure $2 \mathrm{a})$. At low speeds and large $S, R \approx R_{0}$, and a relation between $R_{0}$ and $D_{c}$ can be found for a similar slip-weakening law in which the strength of the fault decays linearly in 
space rather than with slip (Palmer and Rice, 1973),

$$
R_{0}=\frac{9 \pi}{32(1-\nu)} \frac{G D_{c}}{\left(\tau_{p}-\tau_{r}\right)}
$$

where $G$ is the shear modulus and $\nu$ is Poisson's ratio. This can also be expressed in terms of the fracture energy, $\mathcal{G}$, where $\mathcal{G}=D_{c}\left(\tau_{p}-\tau_{r}\right) / 2$. We use eq. (8) to determine the ratio $D_{c} / R_{0}$ implemented in the numerical model and maintain a resolution of 40 elements in $R_{0}$. Rice et al. (2005) estimated that a representative value for $R_{0}$ is 20-40 $\mathrm{m}$ for mid-crustal continental earthquakes.

\subsubsection{Regularized Friction Routine}

We implement a regularized friction routine based on the oblique shock experiments of Prakash and Clifton (1993) and Prakash (1998), who showed that there was no instantaneous response in shear strength to changes in normal stress. The recent laboratory study of Lozos and Kilgore (2010), which repeated the Linker and Dieterich (1992) experiments with improved instrumentation, suggests that no instantaneous change may be the proper interpretation in those too, thus resolving the apparent disagreement with the findings of Prakash and Clifton (1993) and Prakash (1998).

We implement a friction law with a simplified form from that suggested by Prakash and Clifton (1993) and Prakash (1998). For that, the shear strength, $\tau$, evolves over a finite time scale, $t^{*}$, with the relationship

$$
\frac{d \tau}{d t}=-\frac{1}{t^{*}}\left[\tau-f \sigma_{n}\right]
$$

where $\tau(t=0)=f_{s} \sigma_{n}$ and $f$ is generally $f_{s w}$ except in the area of rupture nucleation (see section 3.4). This form was investigated for its stability properties in Ranjith and Rice (2001) for its ability to regularize the ill-posed problem of sliding at constant friction between two dissimilar elastic bodies and implemented in Cochard and Rice (2000) for that case. The bimaterial problem is ill-posed because as the wavelength of the perturbation decreases, the growth rate diverges (Cochard and Rice, 2000; Ranjith and Rice, 2001). While we do not model a material contrast here, use of this law is justified based on observational constraints (Prakash and Clifton, 1993; Prakash, 1998; Lozos and Kilgore, 2010), and it has the added benefit of reducing numerical noise that develops in the ABAQUS contact implementation after the rupture tip passes and sliding at constant friction occurs.

This implementation results is zero instantaneous change in the shear strength 
of the surface in response to an instantaneous change in normal stress (figure 2c). Ideally $t^{*}$ should be much larger than the numerical time step, $\Delta t$, yet very much smaller than the time to undergo slip-weakening, $T$. Here we use, $t^{*}=2 \Delta x / C_{s}$, where $\Delta x$ is the element dimension in the direction of slip. The stable time step can be approximated by $\Delta t=\Delta x / \sqrt{2} C_{p}$. The time to undergo slip-weakening depends on the rupture velocity and is of the order $T=R_{0} / V_{r} \approx 40 \Delta x / C_{s}$, where 40 is determined by our resolution. This results in $t^{*}=2.5 \Delta t$ and $T=20 t^{*}$, but time steps are often smaller than this approximation, and $T$ decreases as the rupture velocity increases. For most models in this study, these parameter choices result in $t^{*} \approx 7.5 \Delta t$ and $T \approx 7 t^{*}$, making this a reasonable choice for $t^{*}$.

We implicitly integrate eq. (9) so that at time step $m, \tau^{m}$ and $\sigma_{n}^{m}$ are related by

$$
\tau^{m}-\tau^{m-1}=-\frac{\Delta t}{t^{*}}\left(\tau^{m}-f^{m} \sigma_{n}^{m}\right)
$$

Then, the shear stress on the fault surface is prescribed as the minimum of $\tau$ (from eq. (10)) and $\tau_{\text {stick }}$ (the stress required to bring the slip velocity to zero at the end of the next time step). In the case of fault opening and eventual re-closure, the stress evolution continues to obey equation (10), but the value of $\tau^{m-1}$ is the shear stress on the surface at the time just before opening.

To understand the effect of using this law, figure 5 shows a direct comparison of the slip and shear stress profiles of a propagating rupture for both the regularized and traditional, non-regularized slip-weakening formulation. The friction values for this comparison are $f_{s}=0.6$ and $f_{d}=0.12$, and an $S$ ratio of $S=1.8$ is used. Both ruptures are traveling at $V_{r}=0.86 C_{s}$ when they cross the observation point (vertical line in figure 5), but the regularized friction law requires a longer crack length to reach the same velocity. The slip distribution is very similar for the two cases, just behind the crack tip, but the regularized case has a larger physical distance over which the shear stress decays from peak to residual values (figure 5b). Although strictly speaking eq. (9) shows that $\tau$ never reaches $\tau_{r}$ in finite time, it does approach $\tau_{r}$ in finite time, the duration of which is set by the value chosen for $t^{*}$.

The time and slip histories at the observation point are shown in figure 6 for the regularized and non-regularized cases. The time history illustrates that, as expected, it takes longer for a point to undergo slip-weakening with the regularized routine due to the non-instantaneous response in shear strength to a change in the friction coefficient. The slip-weakening curves in figure 6 show that while $D_{c}$ is the same for both cases, the effective $D_{c}$ is increased when the regularized friction routine is used. 
This results in a higher fracture energy, $\mathcal{G}$, for the regularized case.

In Appendix A we provide an estimate to this increase in $\mathcal{G}$ for a choice of $t^{*}$ and $V_{r}$. We also show that there is an approximate way to tailor parameter choices (specifically $D_{c}$ ) of the regularized law so as to agree with a desired fracture energy. We note that these parameters depend on the rupture velocity at which the desired fracture energy should be attained. As the rupture velocity increases, there is a Lorentz type contraction of the slip-weakening zone, and therefore the time to undergo slipweakening is reduced. Since we have introduced a time scale through the use of $t^{*}$, and the time to undergo weakening is dependent on the rupture velocity, the specification of regularized routine parameters depends on $V_{r}$ (see equation eq. (A17)).

With the use of the regularized friction routine, the branch activation results can differ from the case of traditional linear slip weakening. However, the small subset of cases that are affected by this choice have initial conditions that are near the transitional regime between branch activation and no branch activation. The general trend of the results (i.e., more or less likely to branch with variation of a given parameter) is not affected by the choice of regularized or non-regularized slip-weakening routines.

\subsection{Rupture Nucleation}

To nucleate rupture we use the forced expansion of a crack (Andrews, 1985; Dunham and Rice, 2008). Nucleation starts by weakening at a point, $x=0$, and forcing the growth of the weakened region by prescribing a non-constant lower coefficient of friction, $f_{e}=f_{e}(x)$, over a growing patch. The weakened patch has a transition of $f_{e}$, from $f_{s}$ (at the edge) to $f_{d}$ (towards the middle), that occurs linearly over a distance $R_{0}$. At the boundaries of the weakened patch, $d f_{e} / d x= \pm\left(f_{s}-f_{d}\right) / R_{0}$, for expansion in the $\pm x$ direction. The edge of the patch expands at a velocity, $V_{e}=0.144 C_{s}$, until the crack approaches an unstable size, and rupture propagates spontaneously. The coefficient of friction due to the expanding patch is determined by

$$
f_{e}(x, t)=\max \left\{f_{s}-\frac{f_{s}-f_{d}}{R_{0}}\left(V_{e} t-|x|\right), f_{d}\right\}
$$

The coefficient of friction on the surface, $f$, is the lesser of $f_{e}$, due to the forced expansion, and $f_{s w}$, which is determined by the amount of slip a point has experienced.

$$
f(x, t)=\min \left\{f_{s w}(s(x, t)), f_{e}(x, t)\right\}
$$

and from this the shear stress on the surface can be determined by using this value 
for friction in regularization routine (see equation eq. (10)).

\section{Results}

\subsection{Conditions for Fault Opening at the Branching Junction}

We investigate under what conditions opening can occur at the branching junction with both the elastic and elastic-plastic material descriptions. This is a physical process, not the result of an algorithmic artifact, that occurs when the stress state on the fault becomes tensile. We will show that opening can, and often does, occur for an elastic material. However, with an elastic-plastic material, opening does not occur except in the case of very high cohesive strength.

\subsubsection{Compressional Side Branches}

First we consider compressional side branches, for which low angles of $\Psi$ are needed for branch activation. For this principal stress orientation, there is a relatively low main fault normal stress to keep the main fault closed. We find that when the branch fault is activated in a purely elastic model, opening occurs on the main fault, prior to the branching junction (figure 7a). The fact that opening can occur is in disagreement with a common modeling procedure (traction-at-split-nodes) that prescribes no fault opening (e.g., Andrews, 1999), although modifications to this procedure can allow for opening (Day et al., 2005). A split-node procedure that assumes no opening results in a fault that supports a tensile normal stress.

Opening occurs in all elastic models with a low $\Psi$, if the full length of the branch ruptures. Opening begins shortly after the rupture tip passes the junction and as slip is accruing on the branch and main faults. Figure $7 \mathrm{a}$ is shown for when the rupture tip is $12 R_{0}$ away on the branch fault and another $4 D_{c}$ of slip has accrued on the main fault. The compressional side of the branch fault is moving up and to the right, resulting in the material pulling away from the main fault. Sometimes, rupture terminates quickly on the branch $\left(L_{s} \leq 3.0 R_{0}\right)$ and only a small amount of slip is able to accrue $\left(s<D_{c}\right)$. In this case of partial branch rupture, no opening occurs, and the default model setup introduces no algorithmic artifacts.

An analogous case is shown in figure $7 \mathrm{~b}$ for an incohesive elastic-plastic material, and we see that opening is inhibited. For this constitutive model, cohesion is negligible, $b=6 \times 10^{-7}\left|\sigma_{y y}^{0}\right|$, the slope of the yield surface was $\mu=0.51$, and a dilation of $\beta=0.256$ was used. All other parameters $\left(S, \Psi, V_{r}\right.$ and $\delta$ ) are equivalent to the elastic 
case, but due to the slower rupture acceleration for the elastic-plastic model, the nucleation points differ. In figure $7 \mathrm{~b}$, the rupture has propagated a distance of $11 R_{0}$ on the branch, but died out on the main fault after $3.3 R_{0}$, and an additional $6 D_{c}$ of slip has occurred on the main fault, prior to the junction.

Figure 8a shows the distribution of plastic deformation for this incohesive model, and it is clear that there is a significant stress concentration at the junction. A spur of substantial plastic deformation, stemming from the triple junction, was the result (note the change in color contour levels between columns 1 and 2 of figure 8). This spur may be related to the conjugate spur noted by Andrews (1989), although that was for an elastic analysis, and was also observed in elastic-plastic analyses of rupture through a kinked fault (Duan and Day, 2008). There is a small amount of plastic deformation on the main fault after the branching junction, due to the continuation of rupture for a finite distance. The rupture propagation on the branch caused only a small amount of plastic deformation around the branch fault, although the width of this zone does increase with propagation distance. The deformed mesh geometry (column 3 of figure 8 ) shows that the fault does not open for this incohesive material description. The stress on the fault, $\sigma_{y y}$, is always negative, indicating that the fault does not undergo tension.

\subsubsection{Effect of Finite Cohesive Strength on Opening}

Compressional side branches, in an elastic model, are prone to opening on the main fault. An incohesive elastic-plastic model can prevent this opening, and here we examine the effect of finite cohesive strengths on the plastic deformation and fault opening, while keeping $\mu$ and $\beta$ constant. Figure 8 shows the plastic deformation for

$6 \times 10^{-7}\left|\sigma_{y y}^{0}\right| \leq b \leq 0.3\left|\sigma_{y y}^{0}\right|$ and a close up of the deformation and deformed mesh geometry at the branching junction. Note that in all of these models, the branch is activated, and rupture terminates on the main fault close to the branching junction. Although these have the same rupture behavior, at the junction the rupture velocity ranges from $0.80 C_{s} \leq V_{r} \leq 0.87 C_{s}$. This is because the nucleation point is the same for all cases shown, and the rupture accelerates faster for larger cohesive strengths, since less plastic deformation occurs.

As the cohesive strength increases, the extent of plastic deformation decreases. This is because stresses far from the fault are not high enough to reach yield when the yield surface has been shifted away from the hydrostatic axis $(\bar{\tau}=0)$. The distribution of plastic deformation in the immediate vicinity of the branching junction (column two of figure 8) is not significantly different as the cohesion changes. There 
are spurs of high deformation stemming from the junction for all cohesive strengths, but the precise size, shape and amount of plastic deformation varies with cohesion. For low cohesion, there is plastic deformation on the entire compressive side of the fault (referring to column 2 of figure 8 only). The only case that exhibits significant opening on either fault is the case of $b=0.3\left|\sigma_{y y}^{0}\right|$ (figure 8e). For slightly less cohesion, $b=0.2\left|\sigma_{y y}^{0}\right|$, opening can occur, but not until long after the rupture has passed the branching junction.

Carmichael (1982) report that cohesive strengths of igneous, sedimentary and metamorphic rock types are in the range $0<c \leq 45 \mathrm{MPa}$, with most values falling below $30 \mathrm{MPa}$ and equally distributed between 0-30 MPa. If a representative normal stress at seismogenic depth is $100 \mathrm{MPa}$, the $b=0.3\left|\sigma_{y y}^{0}\right|$ and $b=0.1\left|\sigma_{y y}^{0}\right|$ models represent a cohesive strength of $c=35 \mathrm{MPa}$ and $11.7 \mathrm{MPa}$, respectively. These two cases are representative of pristine rock, while $b=0.01\left|\sigma_{y y}^{0}\right|$ might approximately represent a highly granulated rock that has regained a minimal amount of cohesive strength through cementation during the interseismic period. Substantial opening occurs if $b=0.3\left|\sigma_{y y}^{0}\right|$, which is at the high end of pristine rock cohesive strength values at depth. For the cohesive strengths that may be reasonable for a mature rock system (figure 8a-c), there is no opening at the junction.

The results for $b=6 \times 10^{-7}\left|\sigma_{y y}^{0}\right|$ can be applied to all depths because the material is effectively incohesive. For larger cohesive strengths, there is a difference between rupture at depth and rupture near the surface. Closer to the surface, where the effective normal stress is lower, $b=0.3\left|\sigma_{y y}^{0}\right|$ would represent a cohesive strength less than that of pristine rock. But high cohesive strengths may not apply to damaged near-surface rocks because observations of exhumed faults indicate a large degree of pulverization (e.g., Chester et al., 2004; Dor et al., 2006). The degree of cementation during the interseismic period will depend on, among other things, the availability of pore fluids and the temperature, so it is not straightforward to quantify the degree of cementation at depth versus near the surface.

\subsubsection{Finite Cohesive Strength with Material Softening}

Opening occurs for the case of $b=0.3\left|\sigma_{y y}^{0}\right|$, which may represent the response of a rock that has regained all of its cohesive strength due to cementation during the interseismic process. We have assumed that the rock has a perfectly plastic response, but softening is a real feature of plastically deforming rocks. This softening occurs as off-fault damage is reactivated due to the high stresses around the rupture tip. This reactivation results in a loss of cohesive strength and the yield surface is shifted 
closer to the hydrostatic axis.

In figure 9 we show the deformation around the branching junction for a range in softening (i.e., negative hardening) values $-0.1 G \leq h \leq 0$. Depending on $h$, the material will lose all cohesive strength after differing amounts of strain. From eq. (5) we see that $d \gamma^{p l}=d b / h$. Therefore, a cohesive strength of $b=0.3\left|\sigma_{y y}^{0}\right|$ will be lost after $1.2 \%$ strain if $h=-0.10 G$ and $12 \%$ strain if $h=-0.01 G$, using the parameters $\left|\sigma_{y y}^{0}\right|=100 \mathrm{MPa}$ and $G=24.3 \mathrm{GPa}$.

The nature of the plastic deformation distribution changes as the material softens. This is due to localization of the plastic deformation, which is in accord with the established theory of Rudnicki and Rice (1975). There is a nonzero critical hardening for the plane strain conditions modeled here, under which localizations can develop. Localization occurs when $h<h_{c r}$, where $h_{c r}$ depends on $\mu, \beta$, and on the ratios of principal stresses. So localizations, for a given stress state, can be inhibited by increasing $h$, or suitably changing $\beta$ or $\mu$. By using $h<0$ we are promoting localizations since as the material deforms, it loses strength, and becomes easier to deform. Due to these localizations, our treatment of the softening is somewhat ad-hoc. We do not have a tractable, unambiguously rigorous methodology for resolving these features (e.g., with strain gradient or non-local features assumed in the stress-deformation constitutive relation, as reviewed in Templeton and Rice (2008)). Thus, aspects of the localized deformations, as we model them, have an inherent grid size dependence.

Of particular importance, regarding the introduction of softening, is that with sufficient softening, opening is inhibited. With softening, the material approaches the incohesisve state as plastic deformation occurs and opening is once again inhibited. With significant softening, $h=-0.1 G$, cohesive strength is quickly lost and opening does not occur. For small amounts of softening, $-0.02 G \leq h \leq 0$, opening of the mesh occurs and is visible to the naked eye (column two of figure 9). For intermediate values of softening $-0.05 G \leq h \leq-0.03 G$, the fault initially opens, but as strain continues to accumulate, and cohesion is lost, the fault opening cannot be sustained and the fault closes.

Hardening $(h>0)$ can cause opening for material parameters that would not otherwise open. For $h=0.03 G$ and $b=0.01\left|\sigma_{y y}^{0}\right|$, the main fault will eventually open a small amount, while with no hardening, it stays closed (figure 8b). By introducing hardening, the material gains cohesive strength as plastic deformation occurs, but this material response does not describe how real damaged material plastically deforms. 


\subsubsection{Branches on the Extensional Side of the Fault}

We also examine the model response for branches that exist on the extensional side of the fault. For an elastic model with $\Psi=47^{\circ}, S=1.0$ and a rupture velocity of $V_{r}=$ $0.80 C_{s}$, both the branch and the main fault are activated. Opening occurs on the main fault further along strike, beyond the branching junction, rather than prior to the junction, as it did for compressional side branches (figure 10a, rupture has propagated $22.9 R_{0}$ past the junction on both faults). The nodes on the branch also come out of contact, but the opening displacement is small and cannot be seen in the image. For the case of compressional side branches, opening begins almost immediately after the branch begins to slip, but for extensional side branches, opening only occurs when a substantial amount of slip $\left(\sim 12 D_{c}\right)$ has accrued on the main fault and rupture is no longer near the junction.

Figure $10 \mathrm{~b}$ and $\mathrm{c}$ shows the stress and plastic deformation for the same $\Psi, S$ and $V_{r}$ as the elastic case, but for an elastic-plastic material. The values for $\mu$ and $b$ are the same as for the compressional side branch, but a larger dilatancy value, $\beta=0.389$, is used here. This larger value inhibits localizations but does not effect the extent of plastic deformation. The rupture is far from the junction, $25 R_{0}$ on both of the faults, and the elastic-plastic deformation inhibits opening at and near the branching junction.

\subsection{Off-fault Plastic Deformation and Branch Activation}

Off-fault plastic deformation changes the branch activation for both compressional and extensional side faults compared to an elastic model (figure 11). With an incohesive material, plasticity can inhibit the activation of compressional side branches and promote activation of extensional side branches. Additionally, with an incohesive material, fault opening does not occur and therefore the results are free from algorithmic artifacts due to the node constraints placed at the triple junction.

\subsubsection{Compressional Side Branches}

Figure 11a shows the change in behavior for a compressional side branch with $S=1.0$, $\Psi=13^{\circ}$ and $V_{r}=0.80 C_{s}$. The branching results are reported with thick lines that denote how far the rupture propagated on each fault. Rupture will propagate the full length of one of the faults, and while the other fault may not fully rupture, a small amount of slip (generally $s<D_{c}$ ) accrues for some distance away from the branching 
junction. If rupture terminates on one of the faults, the distance the fail rupture propagated, $L_{s}$ (normalized by $R_{0}$ ), is reported.

For a purely elastic off-fault behavior, all but the largest branch angle, $\delta=30^{\circ}$, have branch activation, and rupture does not continue on the main fault. For $\delta=30^{\circ}$, a small amount of slip occurs, $s<D_{c}$, but dies out after a distance $L_{s}=0.6$. When the off-fault material behavior is elastic-plastic, with $\beta=0.256$ and negligible cohesion, $b=6 \times 10^{-7}\left|\sigma_{y y}^{0}\right|$, only the $\delta=15^{\circ}$ branch is activated. The rest of the branch fault angles are completely ignored and rupture does not propagate on them for even a short distance $\left(L_{s}=0\right)$.

To determine why these differences occur, we examine the stresses around a propagating crack as well as the stress changes induced on the branch due to rupture propagating past the junction on the main fault. The Coulomb Failure Stress (CFS) accounts for increases in shear stress and decreases in compressional stress, both of which promote failure through the linear combination $\Delta \mathrm{CFS}=\Delta \tau-f_{s} \Delta \sigma_{n}$, where $\tau$ and $\sigma_{n}$ are calculated for a specified fault plane and $\sigma_{n}$ is positive in compression (King et al., 1994).

The branching results in figure 11 are for $V_{r}=0.80 C_{s}$, so this is the rupture velocity used to examine the $\triangle$ CFS distribution. Figure 12a shows the $\triangle$ CFS for both the elastic and the elastic-plastic material model, on all potential fault planes radiating from the rupture tip, at a specified distance, $r$, from the rupture tip. Since the rupture accelerates more quickly in the elastic material, the target rupture velocity is reached for a much shorter crack length, $L$, in the elastic case. For the elastic material, $L=$ $11 R_{0}$, and $r / L=0.034$, while for the elastic-plastic material, $L=33.4 R_{0}$ and $r / L=$ 0.011 . We use the distribution in figure 12 as a qualitative assessment of the influence of elastic-plastic deformation on the stress field. For both the elastic and the elasticplastic scenarios the extensional side of the fault is more highly stressed for failure. There is also a region of the compressional side in which there is an increase in CFS. This region spans a smaller $\theta$ range for the elastic-plastic material, at this $r / L$. It is this increase in CFS that begins to nucleate rupture on a compressional side branch so the small $\theta$ range for the elastic-plastic material leads to less compressional side branch activation.

We also examine the $\triangle \mathrm{CFS}$ on a fictional branch as the rupture propagates on the main fault. The rupture path selection is dependent on the interactions between ruptures on the two faults (Bhat et al., 2007), so by looking at this stressing we see how the main fault rupture alters the stress state on the branch. In figure $12 \mathrm{~b}$ and $\mathrm{c}$ we consider branch angles of $\delta=14^{\circ}$ and $26^{\circ}$. At time 1 , the rupture is at the fictional 
branch junction, and this is when the high stresses associated with the rupture tip nucleate rupture on the branch. At time 2 and 3, the rupture has propagated past the junction a distance of $a$ and $b$ respectively.

For the $14^{\circ}$ branch, at time 1 , both the elastic and elastic-plastic materials result in an increase in CFS at the junction. As the rupture propagates past (times 2 and 3), there are only small differences in the stress distribution (figure 12b). These small differences are consistent with the identical behavior of the elastic and elastic-plastic branch activation results for $\delta=15^{\circ}$. For the $26^{\circ}$ branch, the stress distribution at time 1 is different for the elastic and elastic-plastic cases (figure 12c). The elastic branch has an increase in CFS at the junction, while the elastic-plastic branch has a decrease in CFS. This decrease prevents rupture from ever nucleating on the elasticplastic branch. This is evidenced by the $L_{s}=0.0$ values observed for $\delta=20-30^{\circ}$ (figure 11a) and means that, for an elastic-plastic material, compressional side branches with a large branch angle are unlikely to rupture.

\subsubsection{Extensional Side Branches}

Extensional side faults have a very different response to the inclusion of off-fault plastic deformation. For the elastic cases shown in figure $11 \mathrm{~b}$, with $S=1.4, \Psi=47^{\circ}$ and $V_{r}=0.80 C_{s}$, only the $\delta=-20^{\circ}$ branch is activated. The other branch angles have a small amount of rupture on the branch fault, but rupture terminates quickly. However, when the off-fault material is elastic-plastic, with negligible cohesion and $\beta=0.389$, all of the branch angles investigated show rupture on both the branch and the main fault.

There is also a change in the method of branch activation when an elastic-plastic material is used. For an elastic model, when the main fault rupture tip reaches the junction, rupture nucleates on the branch, at the junction, and propagates unilaterally away. With the elastic-plastic model, rupture tries to nucleate at the branching junction, but only a small pulse of slip travels along the fault, with $s<D_{c}$. After some amount of travel on the branch fault, the slip begins to increase and complete dynamic weakening is attained (figure 13). At this point, rupture propagates away from the junction, but also travels back towards the junction to rupture the entire branch fault. We call this a delayed branch rupture. For $\delta=15^{\circ}$, this occurs $20 R_{0}$ from the branching junction, but for all other branch angles, this happens within a few $R_{0}$ of the junction.

To understand this behavior, we once again examine the stresses on a fictional branch fault as the rupture propagates on the main fault. Figure $12 \mathrm{~d}$ shows that 
there is a big difference between the stress distribution around the propagating crack tip for the elastic $(r / L=0.023)$ and elastic-plastic $(r / L=0.006)$ materials. For the elastic-plastic material, the $\triangle$ CFS is smaller on the extensional side of the fault than on the compressional side. At first glance, this should make it less likely for rupture to initiate on the extensional side elastic-plastic branches, but this disagrees with the observed branch activation.

An analysis of the stress distribution on the branch, as the rupture passes by, explains why the delayed rupture on the branch occurs. At time 1, for the elastic and the elastic-plastic models, both the $\delta=-14^{\circ}$ and $-26^{\circ}$ branches show an increase in CFS over the entirety of the branch. For the elastic case, this is the maximum stressing felt on the branch. But, for the elastic-plastic case, the maximum stressing occurs as the rupture propagates past on the main fault. We believe that it is this maximum peak that is causing the delayed rupture on the branch. We also note that the peak slowly increases in amplitude for the $-14^{\circ}$ case, and can cause the rupture to take off at the long distance from the junction that was observed. The maximum stressing level felt by the compressional branch also occurs as the rupture propagates past the junction (for the elastic-plastic material) but these compressional branch stressing levels are much lower than the stressing levels felt by the extensional side branch. Therefore, delayed rupture of extensional side branches can occur but this same effect does not apply to the compressional side branches considered.

\subsection{Artifacts from Branch Definition in the Finite Element Con- tact Formulation}

There is no fundamental reason, inherent to the FE method, that the FE mesh must be of the form used as our default model setup (figure 3d). An unfortunate feature of standard FE program contact procedures, including ABAQUS, is that only two nodes can be properly represented at the triple junction. This is similar to codes that implement a split-node contact interaction, which is typically written for two node interactions, and therefore cannot handle three nodes at the junction either.

If only two nodes can be present at the branching junction, the two possible configurations are: 1) continuous through the main fault, and 2) continuous through the branch fault. We consider a third configuration for completeness, that has only one node at the junction and neither fault is continuous. We find that each of these possible model configurations results in a different rupture behavior at the branching junction (figure 14). 
We test a few stress states with different $\mathrm{S}$ ratios on the main and branch faults ( $\mathrm{S}=1.4,1.8,2.2$, and 2.6 on the main fault), but maintain a constant orientation of most compressive stress, $\Psi=13^{\circ}$, and rupture velocity, $V_{r}=0.86 C_{s}$, at the branching junction. The branching results are reported in figure 14 .

The default model is shown in figure 14a, where the main fault is defined as a continuous surface. If the main fault does not experience stresses which would cause it to open, this procedure is free of artifacts. On the branch, slip must go to zero as the junction is approached, since the mesh definition prohibits branch slip at the junction. This is how slip must accumulate at the junction if there is no fault opening. Otherwise, right lateral slip between nodes 1 and 2 (figure $3 \mathrm{~b}$ ) would result in opening on the main fault prior to the branching junction (figure 3c). For this model definition, the branch is chosen for low $S$ ratios, and low branch angles, but largely neglected for a large number of stress states and geometries investigated. Opening on the main fault only occurs in the event that the branch fault is completely activated. If rupture is not established on the branch fault, and $s<D_{c}$, there is no fault opening.

The opposite model setup is shown in figure 14c, in which the branch fault is defined as the continuous surface, and the rupture must abruptly stop before continuing to propagate along the main fault. This allows for slip to occur on the branch, at the junction, and opening on the main fault occurs. For this mesh geometry, the branch

is chosen for all stress states and branch geometries investigated, and the main fault is largely ignored, except for a few cases where both faults rupture.

The third case combines the previous two cases and neither fault is continuous through the junction. For this mesh geometry, the behavior is intermediate to the two end members just discussed. The branch is taken more often than when the main fault is continuous, and less frequently than when the branch is the continuous surface.

\section{Discussion}

The fault junction introduces complexities and there are two main points that we will discuss. The first is what is happening physically at the branching junction, and the second is how this can be analyzed numerically. 


\subsection{Does Opening Occur at the Junction?}

If slip occurs only on the main fault, so that the branch is completely neglected, there will be no opening on any faults near the junction. If slip occurs on the branch, at the junction, opening of the main fault must occur (figure 3c). Therefore, in circumstances for which there is no opening of a fault, slip must go to zero on the branch, as the branching junction is approached. This slip distribution on the branch is consistent with the default model setup that we use. Figure 3d shows that for this setup, the elements on the branch, at the junction, share a common node. This forces slip to go to zero at the junction and is free of algorithmic artifacts if no fault opening occurs. When opening does occur, there is no longer a physical basis for the otherwise reasonable algorithmic choice that slip on the branch must vanish at the junction.

However, we have shown that opening can occur if the rupture propagates onto either a compressional or an extensional side fault with a purely elastic material description. For an extensional side branch, this opening is small and does not occur until long after the rupture tip has passed the junction. At the time of opening, rupture has been established on both, or either, of the faults. Therefore, since there is no physical opening until long after the rupture passes, the model results, which indicate on which faults rupture is established, are valid for extensional side branch activation. This is because, in the case of no opening, this FE model definition is free from numerical artifacts.

For a compressional side branch, opening occurs on the main fault, prior to the junction, when the rupture has propagated only a few $R_{0}$ along the branch. If rupture is not established on the branch fault, and $s<D_{c}$, there is no fault opening. The fault only opens if the branch fault is completely activated. We conclude that opening only occurs with sufficient slip on the branch, which would allow the fault to fully weaken. This weakening allows for a self-sustained rupture on the branch, so opening is a result of the activation, not the other way around. While the details of the model (i.e., slip distribution on the main fault at the branch) may not be accurate at long time, the activation or disregard of the branch fault is a valid result.

Fortunately, opening is unlikely to occur at depth, for these geometries, given the fact that stresses around the propagating rupture are high enough to cause plastic deformation (Poliakov et al., 2002). If the fault is mature, the material in the vicinity of the fault should have lost most of its cohesive strength. A non-cohesive material description does not show fault opening for any of the cases examined here, and we discuss why in the next section. If there is cementation during the interseismic period, some cohesive strength may be regained, but even a cohesive strength of 
$b=0.1\left|\sigma_{y y}^{0}\right|$ does not show fault opening. For the highest cohesive strength considered here, $b=0.3\left|\sigma_{y y}^{0}\right|$, which is representative of a strong pristine rock (Carmichael, 1982), opening occurs even with off-fault plastic deformation. But if the high cohesive strength rock softens, which represents the reactivation of initial damage to the rock that was partially healed by cementation, opening may not occur. This softening is a real process that the off-fault material will undergo. We note that our treatment of softening uses a formulation that has material beyond the localization condition and has a somewhat ad-hoc character, with some features of the solution dependent on grid size.

\subsubsection{Stress State in the Presence of Opening}

For the initial stress state used here $\left(\sigma_{z z}^{0}=\left(\sigma_{x x}^{0}+\sigma_{y y}^{0}\right) / 2\right)$, the D-P yield criterion is identical to the M-C yield criterion, and a non-cohesive D-P yield criterion will intersect the origin in the $\tau$ - $\sigma$ space. This yield criterion requires that no principal stresses be tensile. If a segment of the fault has opened, the minimum compressive principal stress, $\sigma_{3}$, goes to zero. As such, Mohr's circle must collapse to a point or it will intersect the yield surface. This means that $\sigma_{1}$ and $\sigma_{2}$ must also go to zero and all stress components must vanish along an opened fault wall. However, if there is cohesion, the yield surface translates up and away from the hydrostatic axis, and a Mohr's circle of non-zero radius (i.e., $\sigma_{1} \neq 0$ ) can exist with $\sigma_{3}=0$.

Now we examine the stress states that satisfy the incohesive D-P yield criterion, eq. (1) with $b=0$. Our model uses the D-P criterion and we determine under what circumstances fault opening can occur. One solution to this relation is that if one principal stress goes to zero, all principal stresses go to zero $\left(\sigma_{1}=\sigma_{2}=\sigma_{3}=0\right)$. To see if this is unique, we also search for a stress state in which yield occurs (the yield criterion is satisfied), and only one principal stress is zero, $\sigma_{3}=0$. If one of the stresses, $\sigma_{2}$, is nonzero, then without loss of generality, we can say that $\sigma_{1}=\lambda \sigma_{2}$, where $\lambda$ can be positive, negative, or zero (note that we have made no assumptions about the relative magnitudes of the principal stresses).

Using this framework, we find that $\lambda$ is only real for $\mu \geq \sqrt{3} / 2=\sin 60^{\circ}$. Since $\mu=$ $\sin \phi$ for the initial stress state, $\lambda$ only exists for $\phi \geq 60^{\circ}$, which does not represent most materials, including those assumed here. Therefore, if $\sigma_{3}=0$, there is no real value for $\lambda$ and D-P can only be satisfied if $\sigma_{2}$ and $\sigma_{1}$ also vanish. For an incohesive constitutive model, if the fault opens, all stresses must vanish along the opened surfaces.

Our results show that no stresses go to zero in the incohesive case, for all configurations studied, and consistently, fault opening does not occur. For the elastic case, 
there are no restrictions on stress state that can be attained, and fault opening does occur without all stresses vanishing. For finite cohesion, the response is intermediate to these two end members, and opening occurs with sufficient cohesion.

\subsection{Numerical Implementation}

The FE method, at its core, is finding displacements of nodes, from which strains and stresses are calculated within the element. Although a system of equations is solved for the nodes, the basic principle of virtual work (including D'Alembert's reversed inertial forces from density times acceleration), does not act on the nodes. Traction boundary conditions are applied to surfaces, and this surface operation is represented by an equivalent, by virtual work, force at a node. There is no fundamental reason why node 1 (figure 3b) cannot have a force applied due to the traction on the branch fault and another force due to traction on the main fault. With both of these forces, the FE equations can be solved. The algorithmic challenge regarding the triple junction arises from the implementation of surface interactions within a given program.

For ABAQUS, and programs with similar treatments of contact interactions, the problem is that a node can only be part of one surface. The surfaces are defined, and then their interactions are prescribed (there is an interaction that defines the main fault and an interaction that defines the branch). An individual node cannot belong to two surfaces, if the two surfaces participate in different contact interactions. This is a result of the contact routine and not an inherent problem with the finite element formulation. In figure 3a, we see that there are three distinct blocks that define the model, and there are five planar surfaces. Three of these surfaces interact as the main fault, and two of these surfaces interact as the branch fault. Within the constraints of the contact routine, we are left with a question regarding nodes 1 and 2 in figure $3 \mathrm{~b}$ : do they belong to the main fault surface or do they belong to the branch surface?

The Traction at Split-Nodes (TSN) procedure is implemented in many numerical models (e.g., Duan and Oglesby, 2005; Day et al., 2005; Ma and Archuleta, 2006). This method, discussed in Andrews (1999), is based on the assumption of two nodes, one on each side of the fault, which only slide past one another and do not open. There are forms of this implementation that do allow for fault opening (Day et al., 2005), but the assumption of two nodes interacting is still the base of the formulation. Since it is assumed that only two nodes are co-located, codes that use this procedure cannot directly implement a geometry in which three nodes exist at the junction. If only two nodes can exist, which node should be removed? 
These two questions, for different implementations, reduce to effectively the same algorithmic issue at the junction. The solution is based on the physical processes that take place at the junction. If rupture propagates onto the branch, and node 1 is allowed to slide up the branch, there will be opening (figure 3c). Therefore if opening does not occur (a common assumption in the TSN implementation), slip on the branch fault must go to zero at the branching junction. This is consistent with our default mesh definition in which nodes 1 and 2 are the same node (figure $3 \mathrm{~d}$ ). And, since there are now only two nodes at the junction, the interactions are no longer ambiguous and this specification can be easily implemented by contact or split-node routines. From this definition we see that the two nodes that now exist at the junction are part of the main fault, and the specification of the contact interactions, in FE models like ABAQUS, is no longer problematic.

Although we investigated the effects of alternate branch definitions (figure 14b \& c), we do not think that these are reasonable setups to use for the fault geometry investigated here. Without one continuous, planar main fault through the model, these alternate definitions force the rupture to abruptly stop on the main fault at the junction and "jump" onto the main fault continuation. If the branch is completely ignored, this results in a strange slip distribution on the main fault, but rupture should be able to continue along the main fault as if the branch was not there.

A final issue worthy of note is the specification of master and slave surfaces in typical contact routines (Hallquist, 2006; ABAQUS Inc., 2007). Master surfaces are defined by elements, and slave surfaces are defined by nodes. Slave nodes cannot penetrate the master surface, but nodes on the master surface can penetrate the slave surface. Due to the large strains that occur at the junction, if the incorrect specification is made, an unreasonable amount of interpenetration is possible. This can be prevented by the specification, for compressional side branches, that the slave surface defines the right hand side of the branch fault in figure 3 .

\section{Conclusions}

When addressing the problem of branched ruptures, it is important to consider both the physical intricacies of the deformation at the branching junction and the algorithmic challenges in properly allowing for them.

Physical opening can occur at the triple junction if the stress state is able to achieve a state of no normal stress across the fault. This is possible and frequently occurs if the off-fault material is elastic. Real rocks are not perfectly elastic, and 
we have shown that an elastic-plastic material description can inhibit opening of the faults near the junction.

There should not be opening at the branching junction unless the rock has a very high cohesive strength. For an incohesive material, intended to account for zones of damaged rock bordering maturely slipped faults, fault opening does not occur. If some cohesive strength is regained due to cementation during the interseismic period, opening does not occur unless there is a full recovery of cohesive strength to pristine rock values. Material softening, which is a real behavior of plastically deforming rocks, represents the reactivation of initial damage. Within the uncertainties of material localization and the inherent grid dependence of these features, we find that sufficient material softening can inhibit opening in cases of high cohesive strength. We conclude that mature fault rocks, even those that have undergone interseismic cementation, should not open.

Besides inhibiting opening at the junction, off-fault plasticity strongly affects the branch activation. Compared to an elastic case, compressional side branches are less likely to activate with a non-cohesive elastic-plastic material description, but extensional side branches are more likely to rupture. The interactions between ruptures on the main fault and the branch are complex, and it is possible to have a delayed rupture on the branch fault driven by the stress field of the rupture propagating on the main fault and its associated plastic deformation.

We also discuss algorithmic issues that may arise due to the presence of such a triple junction. When opening does not occur, the behavior at the triple junction is simplified and standard contact routines in FE programs are able to properly represent the physical situation. A mesh definition in which slip goes to zero on the branch fault as the junction is approached, is the only proper choice if there is no fault opening. This is consistent with the way that we have defined the FE model and results in an easily implemented numerical procedure in other FE and FD models. Thus, in the case of no fault opening, possible artifacts of the finite element methodology do not enter, and we suitably simulate the response of our conceptual model (within limitations of grid refinements and localizations). If fault opening does occur, we show that alternate mesh definitions can drastically change the branch activation results.

For an elastic model, fault opening can occur, but we determine that our numerical algorithms are producing valid results with our specified default geometry. For extensional side branches, opening does not occur until long after the rupture tip has passed the junction. Therefore, since there is no physical opening until after the rupture has been established on the faults, the branch activation results are valid 
for extensional side branches. This is because, in the case of no opening, this FE model definition is free from numerical artifacts. For compressional side branches, opening occurs much earlier than for extensional side branches, but if rupture is not established on the branch fault there is no fault opening. The fault only opens if the branch fault is completely activated. The opening is a result of the activation, not the other way around. While the details of the model (i.e., slip distribution on the main fault at the branch) may not be accurate at long time, the activation or disregard of the branch fault is a valid result.

\section{Data and Resources}

No data were used in this paper. The software package, ABAQUS/Explicit was use to produce model results.

\section{Aacknowledgments}

This research was supported by National Science Foundation (NSF) EAR award 0809610 and by the Southern California Earthquake Center (SCEC); SCEC is funded by NSF Cooperative Agreement EAR-0529922 and USGS Cooperative Agreement 07HQAG0008. The SCEC contribution number for this paper is 1489. We are grateful to Elizabeth Templeton, and Robert Viesca for their initial help in formulating these problems. We also thank the editor and two anonymous reviewers for their helpful comments. 


\section{A Approximation of the Fracture Energy for the Reg- ularized Friction Routine}

In these investigations we used a regularized form of linear slip-weakening which introduces a time scale over which the strength of the surface evolves. Therefore, the time it takes for a point to undergo complete slip-weakening is increased using the regularized routine due to the non-instantaneous response of shear strength to a change in the normal stress. In fact, when the regularized case is used, the time to undergo complete slip-weakening is infinite (see eq. (9)), but the strength closely approaches the residual level after finite time. Using the regularized friction routine rather than the non-regularized slip-weakening law also changes the shape of the slip-weakening curve and thus the fracture energy (figure 6). When $D_{c}$ is the same for both cases, the effective $D_{c}$ is increased in the regularized friction routine. Figure 5 shows that the slip-weakening zone size for the regularized routine, $R^{r}$, is then larger than that of the non-regularized form, $R^{n r}$, where $r$ and $n r$ superscripts denote regularized and non-regularized slip-weakening, respectively.

To choose parameters in the regularized procedure that will simulate a given fracture energy, it must be understood how the choice of regularized time scale, $t^{*}$, and rupture velocity, $V_{r}$, affect the resulting slip-weakening curves.

\section{A.1 Calculation of Fracture Energy}

The time it takes a point to undergo an amount of slip, $D_{c}$, is defined as $T$ (note that for the regularized routine this is not the same as the time is takes for a point to reach a nominal shear stress of $\tau_{r}$ ). $T$ will be different for the regularized and the traditional slip-weakening formulations but our model results show that these do not differ by more than $10 \%$. We define a characteristic average slip velocity during the slip-weakening process, $\bar{V}$, for both regularized $\left(^{r}\right)$ and non-regularized $\left(^{n r}\right)$ forms, by

$$
\overline{V^{r, n r}}=\frac{D_{c}}{T^{r, n r}}
$$

For the non-regularized slip-weakening, the fracture energy, $\mathcal{G}$, is defined in terms of slip, $s$, as

$$
\mathcal{G}=\int_{0}^{D_{c}}\left(\tau(s)-\tau_{r}\right) d s
$$

(Palmer and Rice, 1973) (generalizing Rice (1968) to a case of $\tau_{r} \neq 0$, and for the shear 
mode). The result for $\mathcal{G}^{n r}$ is the familiar

$$
\mathcal{G}^{n r}=\frac{1}{2} D_{c}\left(\tau_{p}-\tau_{r}\right)
$$

and substituting for $D_{c}$ using eq. (A1), this becomes

$$
\mathcal{G}^{n r}=\frac{1}{2} \overline{V^{n r}} T^{n r} \Delta \tau
$$

where $\Delta \tau=\tau_{p}-\tau_{r}$ is the strength drop.

For the regularized case, $\tau \neq \tau(s)$, but rather $\tau=\tau(s, t)$. Therefore to arrive at an approximation to $\mathcal{G}^{r}$ without having to run a numerical model to determine the behavior, we make the approximation that slip accumulates linearly with time as $s=$ $\bar{V} t$. This implies that the slip distribution is linear in space, behind the rupture tip, for steady-state rupture propagation, which we know is not true, but this is a rough approximation. This can change the calculation for fracture energy to an integral over time rather than slip

$$
\mathcal{G}^{r}=\int_{0}^{\infty} \bar{V}\left(\tau(t)-\tau_{r}\right) d t
$$

We substitute equation eq.(7)into eq.(9) and find the form of the strength evolution in time.

$$
\begin{array}{lrl}
\frac{d \tau}{d t}=-\frac{1}{t^{*}}\left[\tau-\left(\tau_{p}-\Delta \tau \frac{\overline{V^{r}} t}{D_{c}}\right)\right] & \text { for } t<T^{r} \\
\frac{d \tau}{d t}=-\frac{1}{t^{*}}\left[\tau-\tau_{r}\right] & \text { for } t \geq T^{r}
\end{array}
$$

Using the initial condition that $\tau=\tau_{p}$ at $t=0$ we find that for $t<T^{r}$

$$
\tau(t)=\tau_{p}-\frac{\Delta \tau}{D_{c}} \overline{V^{r}}\left(t^{*} e^{-t / t^{*}}+t-t^{*}\right)
$$

We define the transitional shear strength, $\tau^{t}$, at time $t=T^{r}$ when an amount of slip, $D_{c}$, has accumulated using eq. (A8)

$$
\tau^{t}=\tau_{p}-\frac{\Delta \tau}{D_{c}} \overline{V^{r}}\left(t^{*} e^{-T^{r} / t^{*}}+T^{r}-t^{*}\right)
$$

Using $\tau^{t}$ as the initial condition for the solution to equation eq. (A7) for $t>T^{r}$, we find

$$
\begin{array}{ll}
\tau(t)=\tau_{p}-\frac{\overline{V^{r}} \Delta \tau}{D_{c}}\left(t^{*} e^{-t / t^{*}}+t-t^{*}\right) \text { for } t<T^{r} \\
\tau(t)=\left(\tau^{t}-\tau^{r}\right) e^{\left(T^{r}-t\right) / t^{*}}+\tau^{r} \quad \text { for } t \geq T^{r}
\end{array}
$$


¿From here we evaluate the fracture energy for the regularized procedure by substitution of eq. (A10)-eq. (A11) into eq.(A5).

$$
\mathcal{G}^{r}=\frac{\Delta \tau \overline{V^{r}} T^{r}}{2}\left(1+\frac{2 t^{*}}{T^{r}}\right)
$$

\section{A.1.1 Ratio of Fracture Energies}

Using the fact that $\overline{V^{r}} T^{r}=\overline{V^{n r}} T^{n r}=D_{c}$, the ratio of fracture energies, $\mathcal{G}$, for the regularized and non-regularized linear slip-weakening is estimated from eq. (A4) and eq. (A12) to be

$$
\mathcal{G}=\frac{\mathcal{G}^{r}}{\mathcal{G}^{n r}}=1+\frac{2 t^{*}}{T^{r}}
$$

To utilize this relation, an estimate is needed for $T^{r}$. $T^{r}$ is not significantly different from $T^{n r}$, and $T^{n r}$ can be estimated as

$$
T^{n r}=\frac{R^{n r}}{V_{r}}
$$

and where $R^{n r}$ is found from the equation

$$
\frac{R^{n r}}{R_{0}}=\frac{1}{f\left(V_{r}\right)}
$$

where

$$
f\left(V_{r}\right)=\frac{\alpha_{s} V_{r}^{2}}{(1-\nu) C_{s}^{2}\left(4 \alpha_{s} \alpha_{p}-\left(1+\alpha_{s}^{2}\right)^{2}\right)}
$$

is the familiar equation from Rice (1980) and $\alpha_{p}^{2}=1-V_{r}^{2} / C_{p}^{2}$ and $\alpha_{s}^{2}=1-V_{r}^{2} / C_{s}^{2}$.

Using eq. (A14)-eq. (A16), we can arrive at an approximate value for $T^{n r}$, and predict $\mathcal{G}$ using eq. (A13) for a specified $t^{*}$ and $V_{r}$. We compare this to the numerically determined $\mathcal{G}$ values, $\mathcal{G}_{\text {meas }}$, in figure 15 . This comparison used three values for $t^{*}$ $\left(t^{*}=1,2\right.$, and $\left.4 \Delta x / C_{s}\right)$, and a range in rupture velocities $\left(V_{r}=0.42 C_{s}-0.90 C_{s}\right), \mathrm{S}$ ratios $(S=1.0-3.0)$, and residual friction coefficients $\left(f_{s}=0.12-0.4\right)$.

Using the above equations consistently under-predicts the measured ratio. The discrepancy is due to the assumption in the above derivation that the slip accumulates linearly with time, which we show to be an overly simple assumption in figure 6. But, with a simple modification, and using a value for $T^{n r}$ that is $70 \%$ of the value calculated from eq. (A14)-eq. (A16), a good approximation to the resulting fracture energy can be made (pink line in figure 15).

A desired fracture energy is represented by a $D_{c}$ in the non-regularized procedure. 
To represent this same fracture energy within the regularized routine, at a specified rupture velocity, a modified value for $D_{c}$ must be used. The above relations can be use to find this modified $D_{c}$

$$
D_{c}^{\text {mod. }}=\frac{D_{c}}{2+\frac{4 t^{*}}{0.7 T^{n r}}}
$$

where $T^{n r}$ is calculated for the desired $V_{r}$ using eq. (A14)-eq. (A16) and the factor of 0.7 is the corrective factor.

The ratio of fracture energies changes with the rupture velocity. As the rupture velocity increases, there is a Lorentz type contraction of the slip-weakening zone, imbedded in the relation eq. (A15), and therefore the time to undergo slip-weakening is reduced as the rupture velocity is increased. Since we have introduced a time scale through the use of $t^{*}$, and the time scale for weakening is dependent on the velocity, there will be a non-constant ratio of fracture energies that's dependent on the rupture velocity. 


\section{References}

ABAQUS Inc. (2007), ABAQUS Theory manual, Version 6.7, Providence, R. I.

Ando, R., and T. Yamashita (2007), Effects of mesoscopic-scale fault structure on dynamic earthquake ruptures: Dynamic formation of geometrical complexity of earthquake faults, J. Geophys. Res., 112(B9), doi:10.1029/2006JB004612.

Ando, R., B. E. Shaw, and C. H. Scholz (2009), Quantifying natural fault geometry: Statistics of splay fault angles, Bull. Seis. Soc. Am., 99(1), 389-395, doi: $10.1785 / 0120080942$.

Andrews, D. J. (1976), Rupture velocity of plane strain shear cracks, J. Geophys. Res., 81(32), 5679-5687.

Andrews, D. J. (1985), Dynamic plane-strain shear rupture with a slip-weakening friction law calculated by a boundary integral method, Bull. Seis. Soc. Am., 75(1), $1-21$.

Andrews, D. J. (1989), Mechanics of fault junctions, J. Geophys. Res., 94(B7), 93899397.

Andrews, D. J. (1999), Test of two methods for faulting in finite-difference calculations, Bull. Seis. Soc. Am., 89(4), 931-937.

Aochi, H., E. Fukuyama, and M. Matsuura (2000), Selectivity of spontaneous rupture propagation on a branched fault, Geophys. Res. Lett., 27(22), 635-638.

Bhat, H. S., R. Dmowska, J. R. Rice, and N. Kame (2004), Dynamic slip transfer from the Denali to Totschunda faults, Alaska: testing theory for fault branching, Bull. Seis. Soc. Am., 94(6), S202-S213, doi:10.1785/0120040601.

Bhat, H. S., M. Olives, R. Dmowska, and J. R. Rice (2007), Role of fault branches in earthquake rupture dynamics, J. Geophys. Res., 112(B11), doi: 10.1029/2007JB005027.

Carmichael, R. S. (1982), Handbook of Physical Properties of Rocks, CRC Press, Boca Raton, FL.

Chester, F. M., J. S. Chester, D. L. Kirschner, S. E. Schulz, and J. P. Evans (2004), Structure of large-displacement strike-slip fault zones in the brittle continental 
crust, in Rheology in the LIthosphere at Continental Margins, edited by G. D. K. B. T. N. W. Driscoll and D. L. Kohlstedt, Columbia Univ. Press, New York.

Cochard, A., and J. R. Rice (2000), Fault rupture between dissimilar materials: Ill-posedness, regularization and slip-pulse response, J. Geophys. Res., 105(B11), 25,891-907.

Day, S. M., L. A. Dalguer, N. Lapusta, and Y. Liu (2005), Comparison of finite difference and boundary integral solutions to three-dimensional spontaneous rupture, $J$. Geophys. Res., 110, B12,307, doi:10.1029/2005JB003813.

Dor, O., Y. Ben-Zion, T. K. Rockwell, and J. Brune (2006), Pulverized rocks in the Mojave section of the San Andreas Fault Zone, Earth. Planet. Sci. Lett., 245, 642654.

Duan, B., and S. M. Day (2008), Inelastic strain distribution and seismic radiation from rupture of a fault kink, J. Geophys. Res., 113, B12,311, doi: 10.1029/2008JB005847.

Duan, B., and D. D. Oglesby (2005), Multicycle dynamics of nonplanar strike-slip faults, J. Geophys. Res., 110, B03,304, doi:10.1029/2004JB003298.

Duan, B., and D. D. Oglesby (2007), Nonuniform prestress from prior earthquakes and the effect on dynamics of branched fault systems, J. Geophys. Res., 112, B05,308, doi:10.1029/2006JB004443.

Dunham, E. M., and J. R. Rice (2008), Earthquake slip between dissimilar poroelastic materials, J. Geophys. Res., 113, B09,304, doi:10.1029/2007JB005405.

Fliss, S., H. S. Bhat, R. Dmowska, and J. R. Rice (2005), Fault branching and rupture directivity, J. Geophys. Res., 110(B6), doi:10.1029/2004JB003368.

Fukuyama, E., and T. Mikumo (2006), Dynamic rupture propagation during the 1891 Nobi, central Japan, earthquake: A possible extension to the branched faults, Bull. Seis. Soc. Am., 96(4A), 1257-1266, doi:10.1785/01200050151.

Hallquist, J. O. (2006), LS-DYNA Theory manual, Livermore, CA.

Harris, R. A., and S. M. Day (1999), Dynamic 3D simulations of earthquakes on en echelon faults, Geophys. Res. Lett., 26(14), 20892092. 
Ida, Y. (1972), Cohesive force across the tip of a Longitudinal-Shear crack and Griffith's specific surface energy, J. Geophys. Res., 77(20), 3796-3805.

Kame, N., J. R. Rice, and R. Dmowska (2003), Effect of prestress state and rupture velocity on dynamic fault branching, J. Geophys. Res., 108(B5), 2265, doi:10.1029/2002JB002189.

King, G. C. P., R. S. Stein, and J. Lin (1994), Static stress changes and the triggering of earthquakes, Bull. Seis. Soc. Am., 84(3), 935-953.

Linker, M. F., and J. H. Dieterich (1992), Effects of variable normal stress on rock friction: Observations and constitutive relations, J. Geophys. Res., 97, 4923-4940.

Lozos, J. C., and B. Kilgore (2010), Laboratory observations of the response of fault strength as normal stress is changed, and implications for dynamic rupture, SCEC Annual Meeting, Proceedings and Abstracts, XX, 2-076.

Lysmer, J., and R. L. Kuhlemeyer (1969), Finite dynamic model for infinite media, $J$. Eng. Mech. Div. ASCE, pp. 859-877.

Ma, S., and R. J. Archuleta (2006), Radiated seismic energy based on dynamic rupture models of faulting, J. Geophys. Res., 111, B05,315, doi:10.1029/2005JB004055.

McKenzie, D. P., and W. J. Morgan (1969), Evolution of triple junctions, Nature, 224, $125-133$.

Oglesby, D. D., S. M. Day, Y.-G. Li, and J. E. Vidale (2003), The 1999 Hector Mine earthquake: The dynamics of a branched fault system, Bull. Seis. Soc. Am., 93(6), $2459-2476$.

Palmer, A. C., and J. R. Rice (1973), Growth of slip surfaces in progressive failure of over-consolidated clay, Proceedings of the Royal Society of London Series A-Mathematical Physical and Engineering Sciences, 332(1591), 527-548.

Poliakov, A. N. B., R. Dmowska, and J. R. Rice (2002), Dynamic shear rupture interactions with fault bends and off-axis secondary faulting, J. Geophys. Res., 107(B11), 2295, doi:10.1029/2001JB000572.

Prakash, V. (1998), Frictional response of sliding interfaces subjected to time varying normal pressures, J. of Tribol., 120, 97-102, doi:10.1115/1/2834197. 
Prakash, V., and R. J. Clifton (1993), Time resolved dynamic friction measurements in pressure-shear, in Experimental Techniques in the Dynamics of Deformable Solids, edited by A. K. T. Ramesh, pp. 33-48, Appl. Mech. Div., Am. Soc. of Mechanical Eng., Ney York.

Ranjith, K., and J. R. Rice (2001), Slip dynamics at an interface between dissimilar materials, J. Mech. Phys. Solids, 49, 341-361.

Rice, J. R. (1968), A path independent integral and the approximate analysis of strain concentration by notches and cracks, J. App. Mech., 35, 379-386.

Rice, J. R. (1980), The mechanics of earthquake rupture, in Physics of the Earth's Interior, edited by A. M. Dzieswonski and E. Boschi, Italian Physical Society and North Holland Publ. Co., Amsterdam.

Rice, J. R., C. G. Sammis, and R. Parsons (2005), Off-fault secondary failure induced by a dynamic slip pulse, Bull. Seis. Soc. Am., 95(1), 109-134, doi: $10.1785 / 0120030166$.

Rudnicki, J. W., and J. R. Rice (1975), Conditions for localization of deformation in pressure-sensitive dilatant materials, Journal of the Mechanics and Physics of Solids, 23, 371-394.

Scholz, C. H., R. Ando, and B. E. Shaw (2010), The mechanics of first order splay faulting: The strike-slip case, J. Struct. Geol., 32, 118-126, doi:10.1016/j.jsg.2009.10.007.

Sowers, J. M., J. R. Unruh, W. R. Lettis, and T. D. Rubin (1994), Relationship of the Kickapoo fault to the Johnson Valley and Homestead Valley faults, San Bernardino County, California, Bull. Seis. Soc. Am., 84(3), 528-536.

Templeton, E. L., and J. R. Rice (2008), Off-fault plasticity and earthquake rupture dynamics: 1. Dry materials or neglect of fluid pressure changes, J. Geophys. Res., 113, B09,306, doi:10.1029/2007JB005529.

Templeton, E. L., A. Baudet, H. S. Bhat, R. Dmowska, J. R. Rice, A. J. Rosakis, and C. Ernst Rousseau (2009), Finite element simulations of dynamic shear rupture experiments and dynamic path selection along kinked and branched faults, J. Geophys. Res., 114, B08,304, doi:10.1029/2008JB006174.

Templeton, E. L., H. S. Bhat, R. Dmowska, and J. R. Rice (2010), Dynamic rupture through a branched fault configuration at Yucca Mountain, and resulting ground motions, Bull. Seis. Soc. Am., 100(4), 1485-1497, doi:10.1785/0120090121. 


\section{Author Affiliation and Addresses}

Nora DeDontney

Dept. of Earth and Planetary Sciences

20 Oxford St.

Harvard University

Cambridge MA 02138, USA

(ndedontn@post.harvard.edu)

James R. Rice

Dept. of Earth and Planetary Sciences and School of Engineering and Applied Sciences

29 Oxford St.

Harvard University

Cambridge MA 02138, USA

Renata Dmowska

School of Engineering and Applied Sciences

29 Oxford St.

Harvard University

Cambridge MA 02138, USA 


\section{Tables}

Table 1: Representative Material Parameters

\begin{tabular}{lll}
\hline$\rho$ & Density & $2700 \mathrm{~kg} / \mathrm{m}^{3}$ \\
$E$ & Young's modulus & $60.84 \mathrm{GPa}$ \\
$G$ & Shear modulus & $24.34 \mathrm{GPa}$ \\
$\nu$ & Poisson's ratio & 0.25 \\
$C_{p}$ & P wave speed & $5200 \mathrm{~m} / \mathrm{s}$ \\
$C_{s}$ & S wave speed & $3002 \mathrm{~m} / \mathrm{s}$ \\
$R_{0}$ & Slip weakening zone size & $40 \mathrm{~m}$ \\
$f_{s}$ & Static friction & 0.6 \\
$f_{d}$ & Dynamic friction & 0.12 \\
$D_{c}$ & Slip weakening distance & $\sim 70 \mathrm{~mm}$ \\
$t^{*}$ & Regularization time scale & $6.66 \mathrm{e}-4 \mathrm{~s}$ \\
$\left|\sigma_{y y}^{0}\right|$ & Normal stress & $100 \mathrm{MPa}$ \\
$b$ & Drucker-Prager cohesion & $60 \mathrm{~Pa}-30 \mathrm{MPa}$ \\
$\mu$ & Drucker-Prager surface slope & 0.51 \\
$\beta$ & Plastic dilatancy & $0.257-0.389$ \\
\hline
\end{tabular}




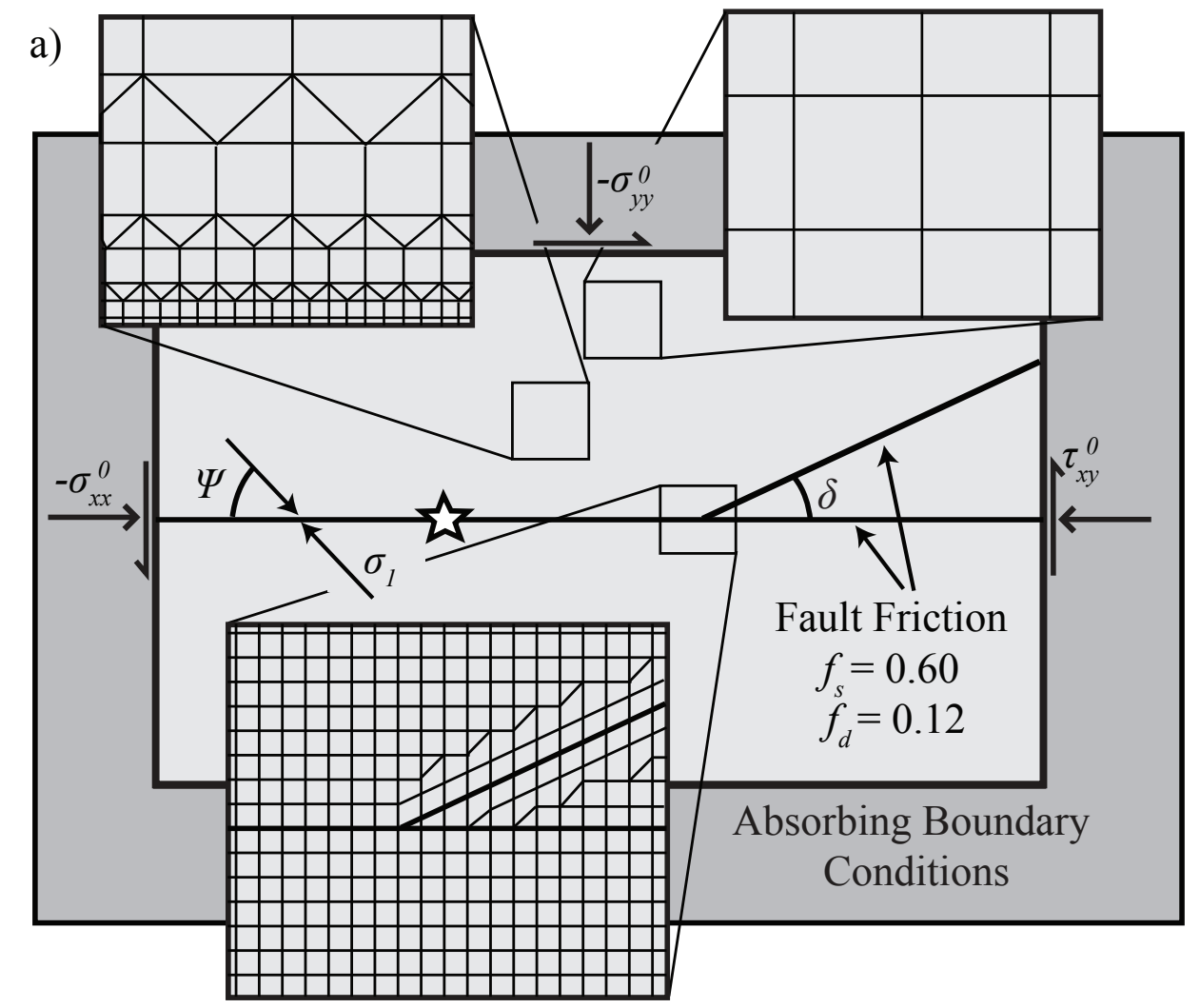

b)

Compressional

Side Branch

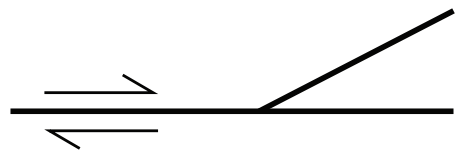

Extensional

Side Branch

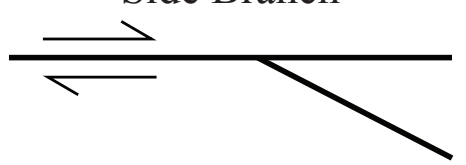

Figure 1: (a) Basic Finite Element Model setup with absorbing boundary conditions and a uniform stress state with plane strain elements. New mesh geometry in which larger elements are utilized far away from the faults for computational efficiency. The few corner elements which are much smaller than the fine resolution section are artificially denser so as to not dictate the model time step. (b) Definition of compressional and extensional side branches. 


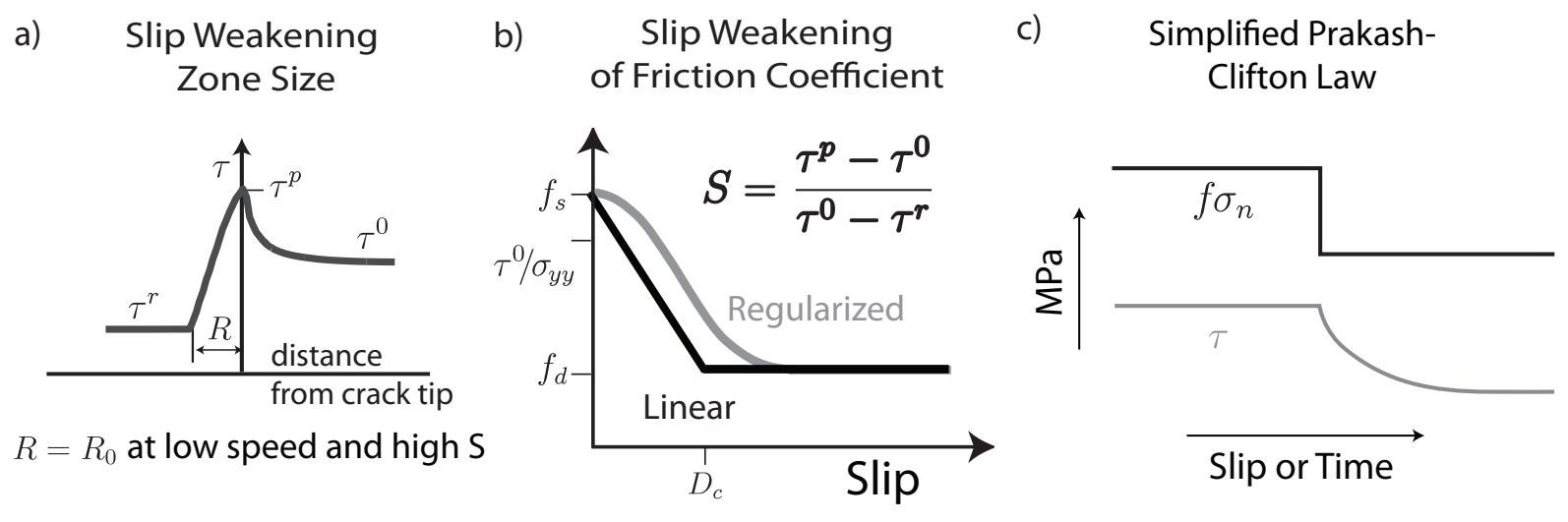

Figure 2: Slip weakening laws. (a) $R$ is the physical dimension over which stress decays from peak to residual value. (b) For linear slip weakening, $D_{c}$ is the amount of slip over which the strength decays from peak to residual value. For the regularized friction routine, this amount of slip is increased. (c) Regularized friction routine has a non-instantaneous response in strength (gray line) to an instantaneous change in friction or normal stress (black line).

a)

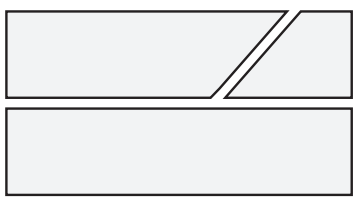

c)

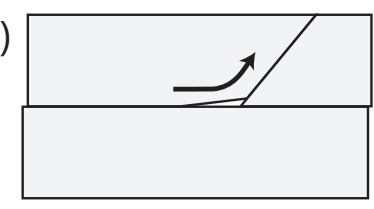

b)

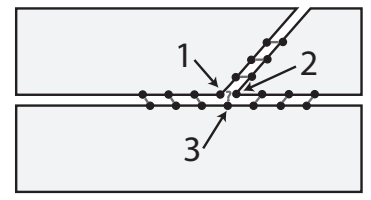

d)

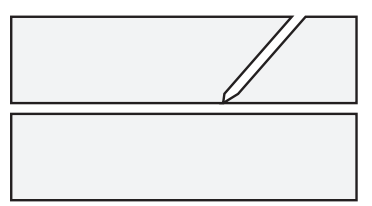

Figure 3: Models used for discussion of branch point definition. (a) Fault bounded blocks. (b) Node discretization and surface interactions. (c) Junction opening. (d) Model geometry used. 


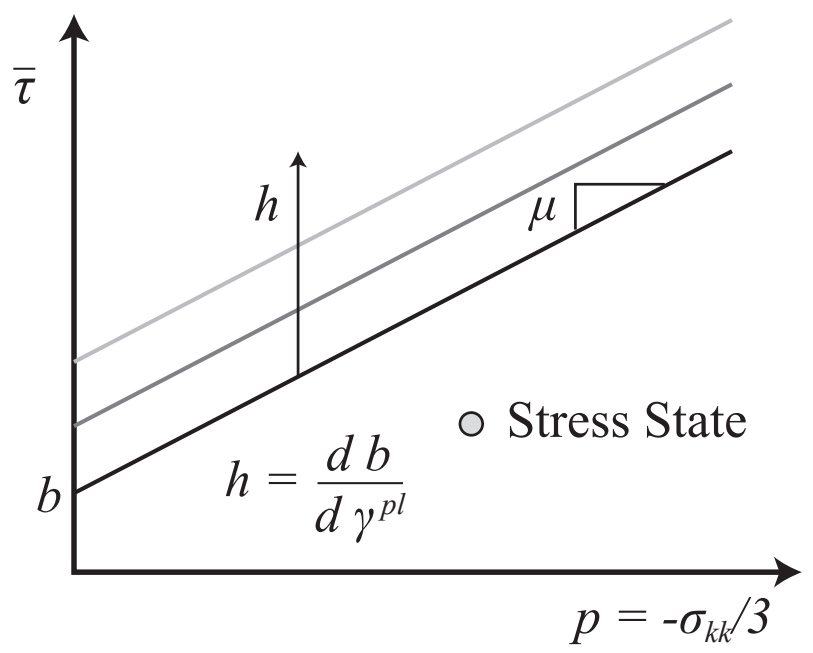

Figure 4: The Drucker-Prager yield surface is defined by a cohesion, $b$, and a slope, $\mu$. Hardening, $h$, shifts the yield surface as plastic deformation, $\gamma^{p l}$, occurs. The initial stress state is represented by a point in this space. 

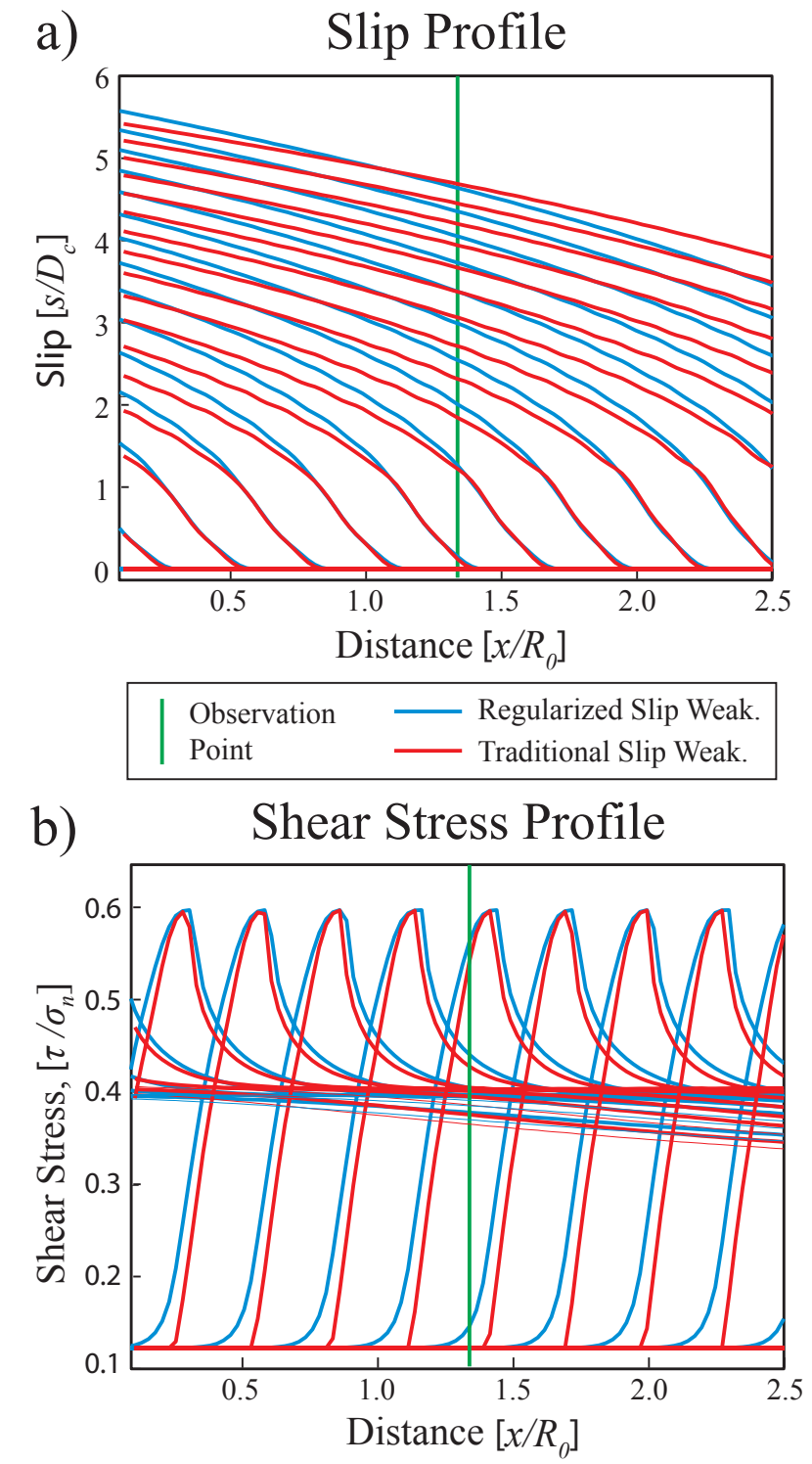

Figure 5: Comparison of the regularized slip friction law used in this study to the non-regularized slip-weakening law. Lines plotted at even time increments. Vertical line denotes the position at which the friction history is reported in figure 6. (a) The slip distribution is similar, near the rupture tip, for the two laws. (b) The regularized routine results in a larger physical distance over which slip-weakening occurs. 

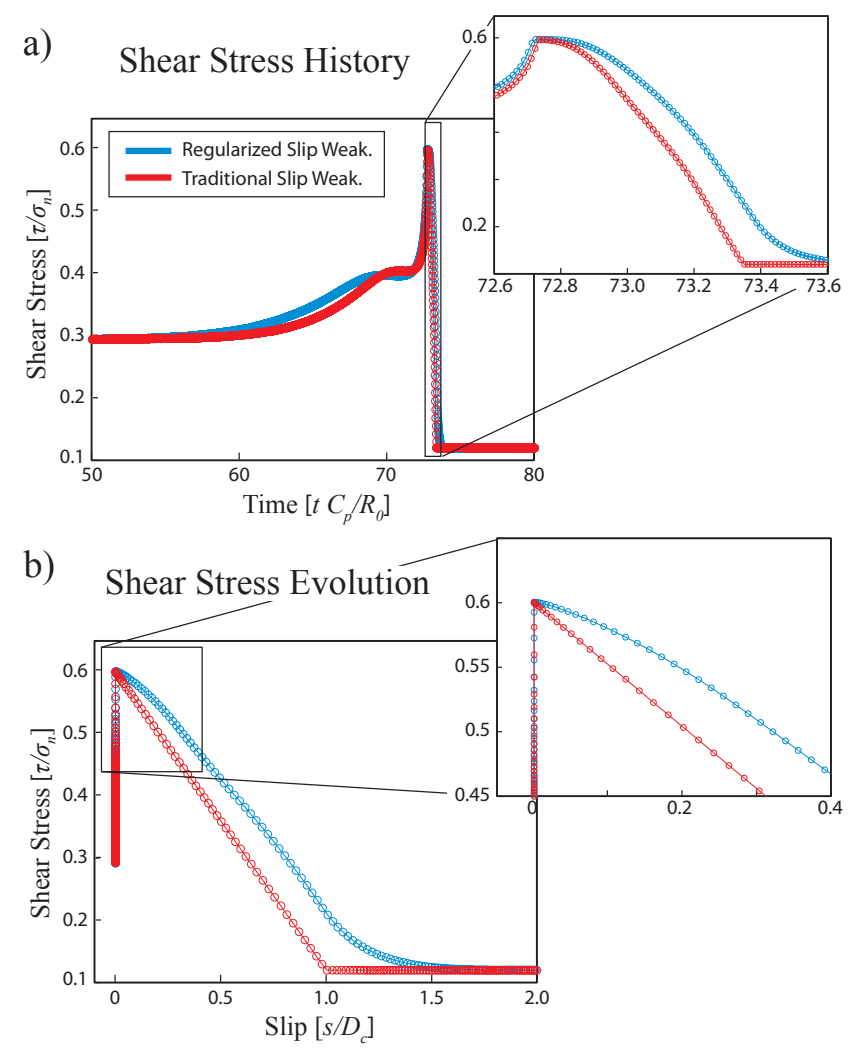

Figure 6: Shear stress values at a point, as the rupture tip travels at $V_{r}=0.86 C_{s}$. Regularized friction effectively increases the slip-weakening distance. (a) Shear stress time history of the observation point. Note that the time histories have been offset so that the peaks align. (b) Shear stress evolution as slip accumulates, i.e. the slipweakening curve. 


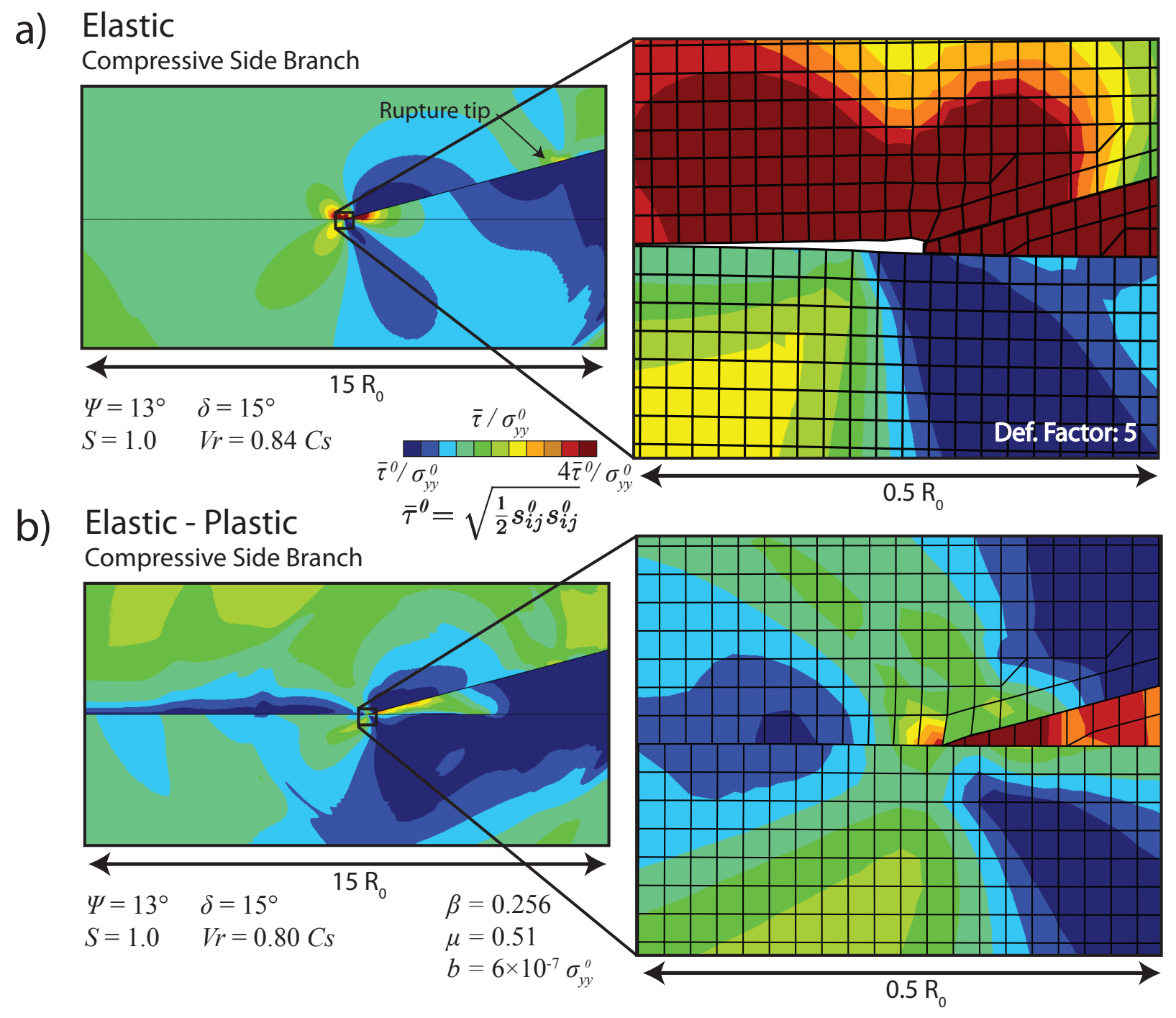

Figure 7: Image of the branching junction some time after the rupture has propagated onto the compressive side branch and a small distance along the main fault. Contours show the shear stress level, $\bar{\tau}^{0} / \sigma_{y y}^{0}=0.82$. (a) Opening occurs at the junction for an elastic off-fault material. (b) Opening does not occur for an incohesive elastic-plastic material. 

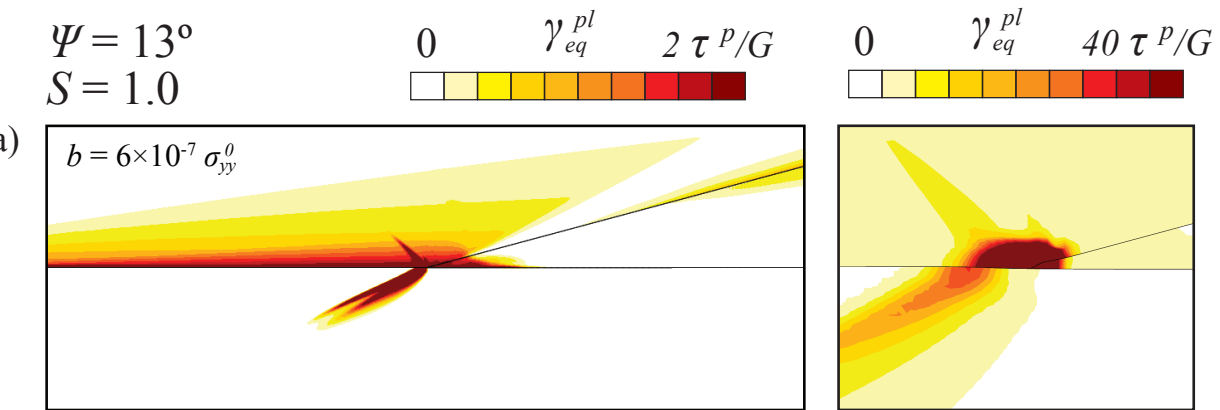

b)
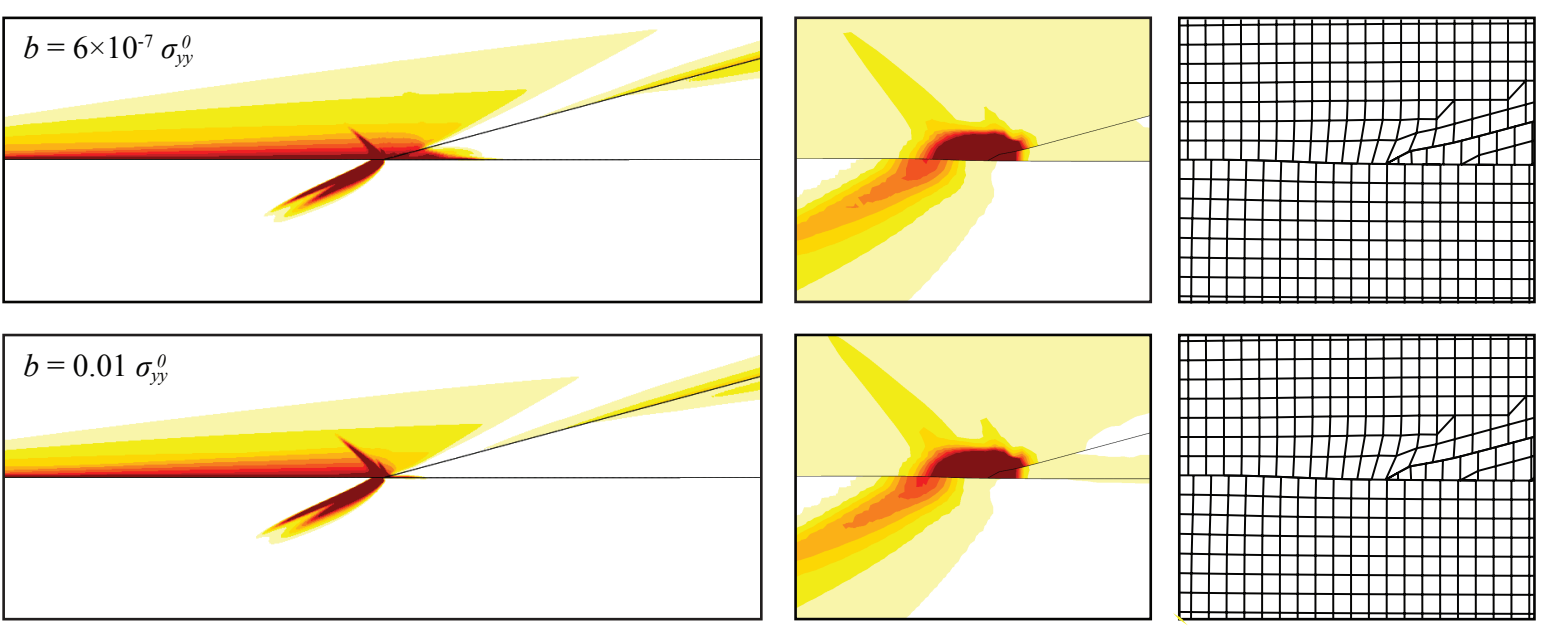

c)
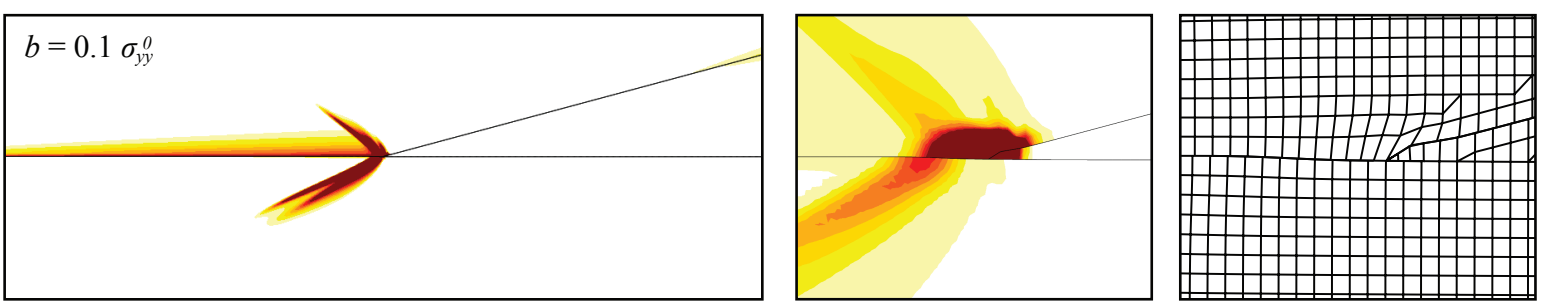

d)
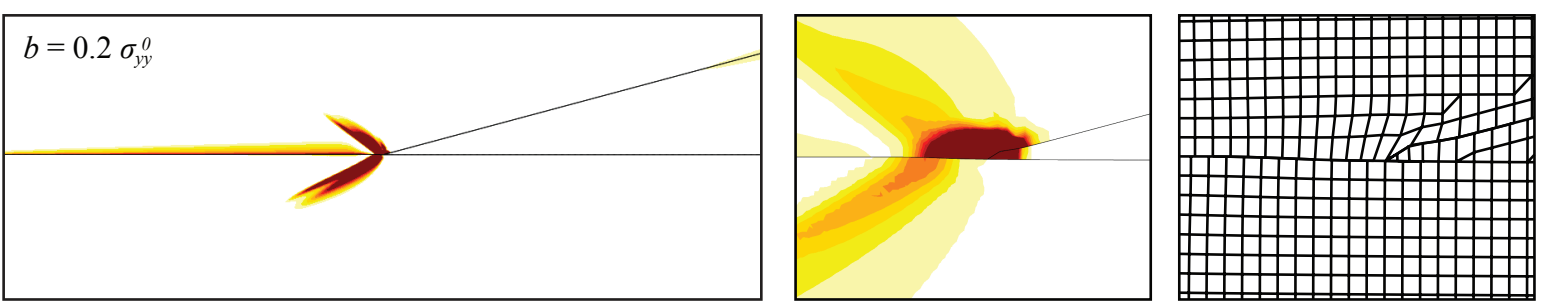

e)
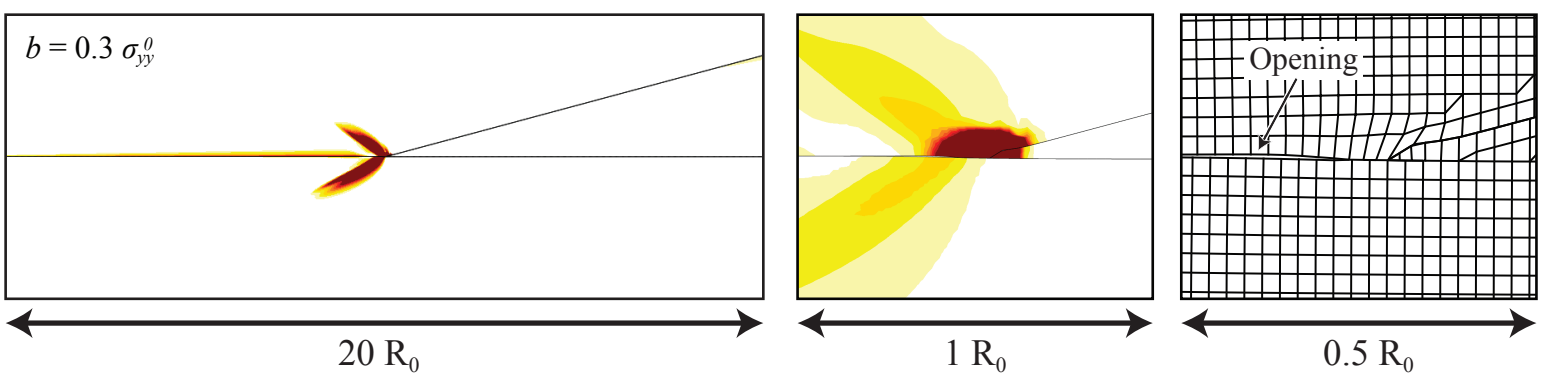

Figure 8: Plastic deformation for models with varying amounts of cohesion, increasing from (a) to (d). Column two is the same case as shown in column one, but shows a close up of the branching junction and uses a different color scale since deformation is very high near the junction. Column three shows the mesh deformation for a further close up of the junction. (d) The only case that shows opening on the main fault. 


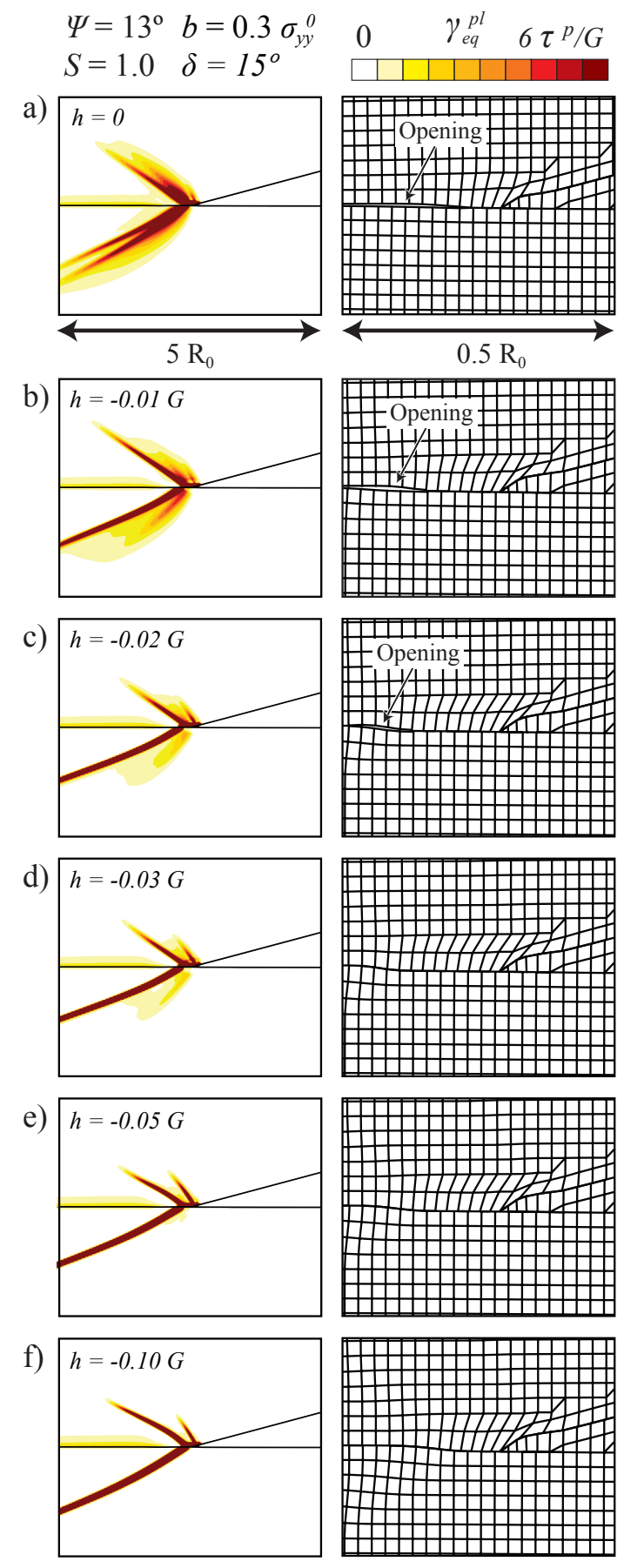

Figure 9: The addition of material softening to the strongest cohesion case shown in figure 8a only affects the plastic strain in the area of the branching junction. Here we show the response for increasing amounts of softening which reduces the cohesive strength and allows the plastic shear strain to localize. With sufficient softening, fault opening no longer occurs. Softening is a real feature of plastically deforming rocks, but the implementation here does have an inherent grid size dependence. 
a) Elastic

Extensional Side Branch

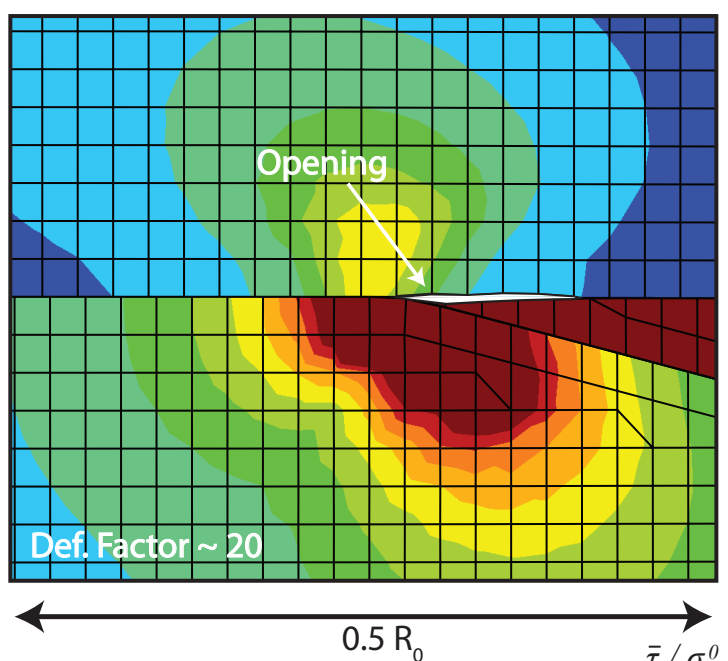

$\Psi=47^{\circ} \quad \delta=-15^{\circ}$

$S=1.0 \quad V r=0.80 C_{s}$
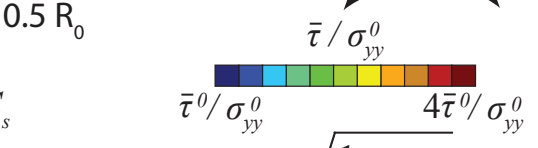

$$
\bar{\tau}^{0}=\sqrt{\frac{1}{2} s_{i j}^{0} s_{i j}^{0}}
$$

b) Elastic - Plastic

Extensional Side Branch

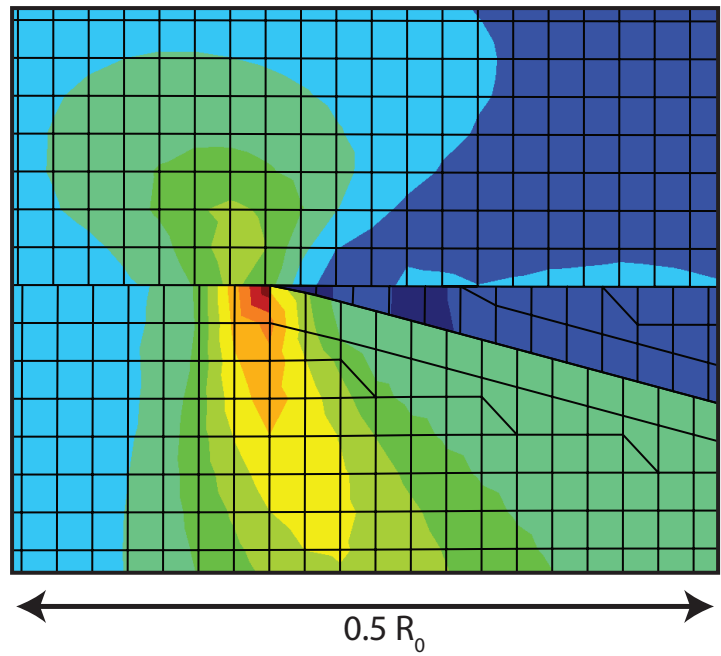

$\Psi=47^{\circ} \quad \delta=-15^{\circ} \quad \beta=0.389$

$S=1.0 \quad V r=0.80 C_{s} \quad \mu=0.51$

$b=6 \times 10^{-7} \sigma_{y y}^{0}$

C) Elastic - Plastic Extensional Side Branch

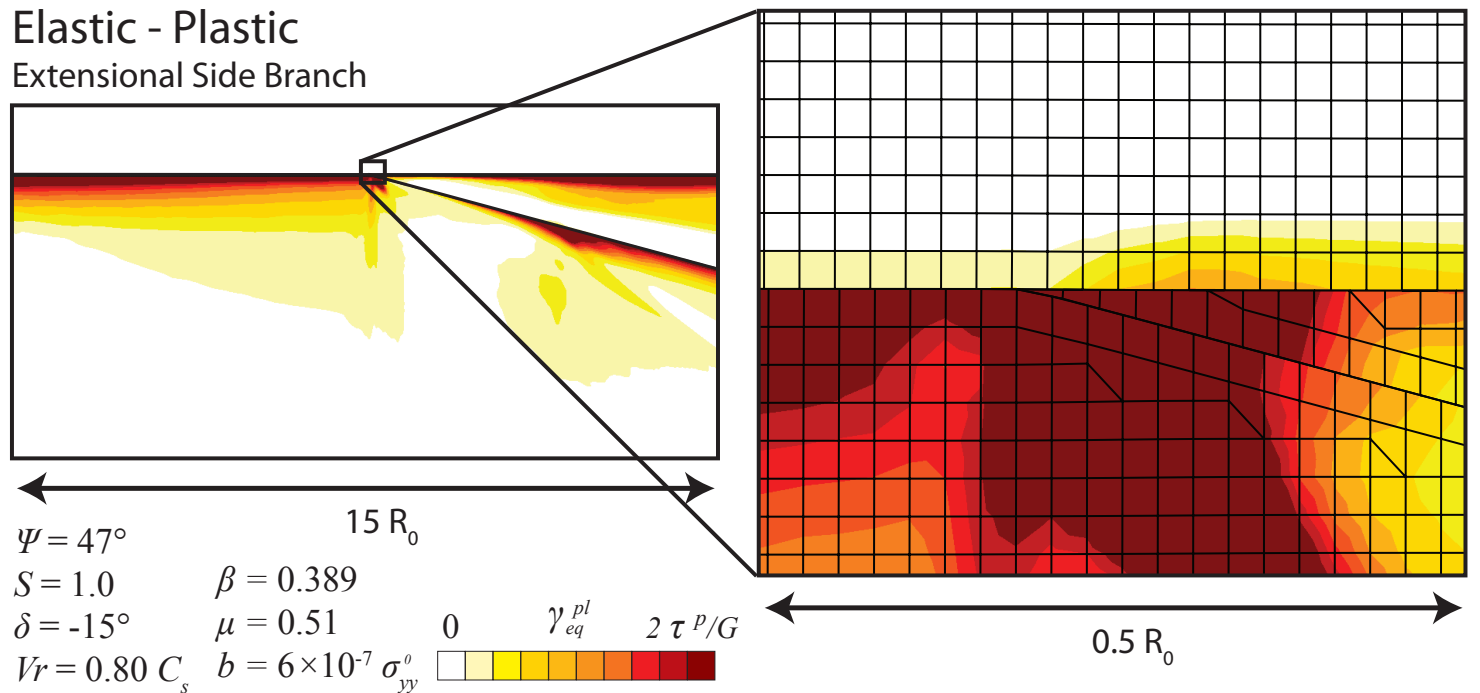

Figure 10: Image of the branching junction some time after the rupture has propagated onto the extensional side branch and a small distance along the main fault. (a) Opening occurs at the junction for elastic cases. Vertical deformation is exaggerated by a deformation factor of 20 , only on the main fault to make the small opening visible. (b) \& (c) Opening does not occur at the junction with an incohesive elastic-plastic material behavior. 
a)

$$
\Psi=13^{\circ}
$$

$$
S=1.0, V_{r}=0.8 C_{s}
$$

\section{Elastic}

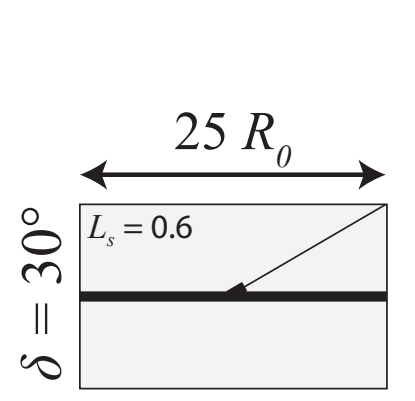

$$
\beta=0.256
$$

$$
\mu=0.51
$$
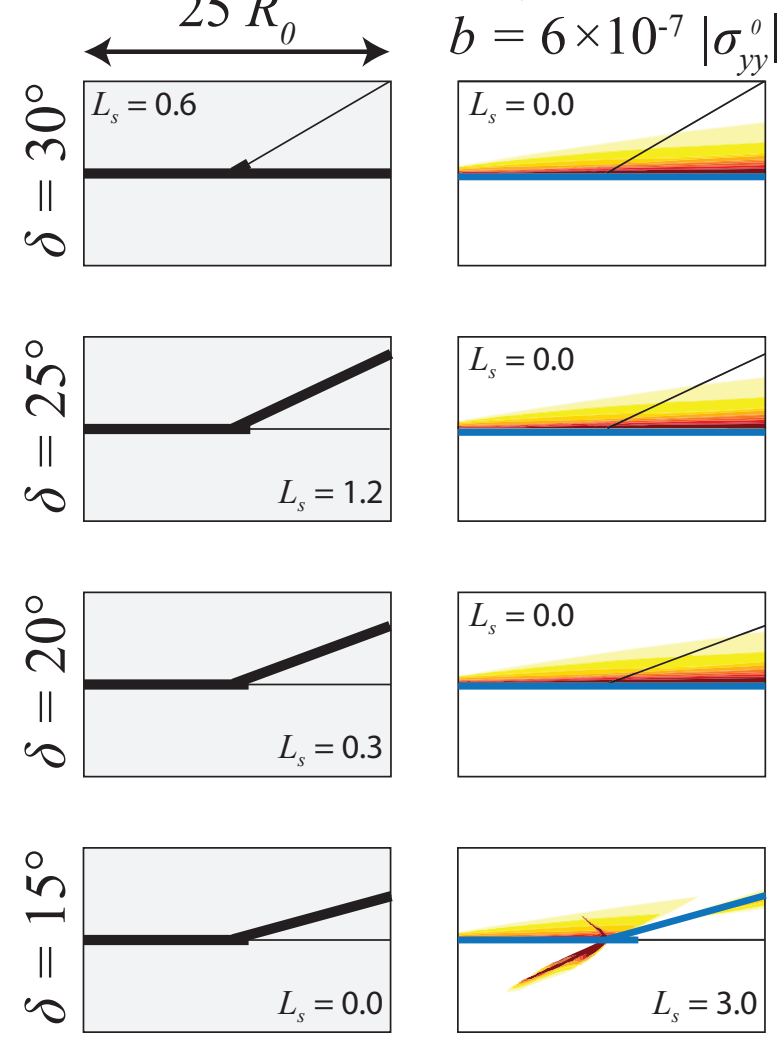

0 b)

$$
\begin{gathered}
\Psi=47^{\circ} \\
S=1.4, V_{r}=0.8 C_{s}
\end{gathered}
$$

\section{Elastic}
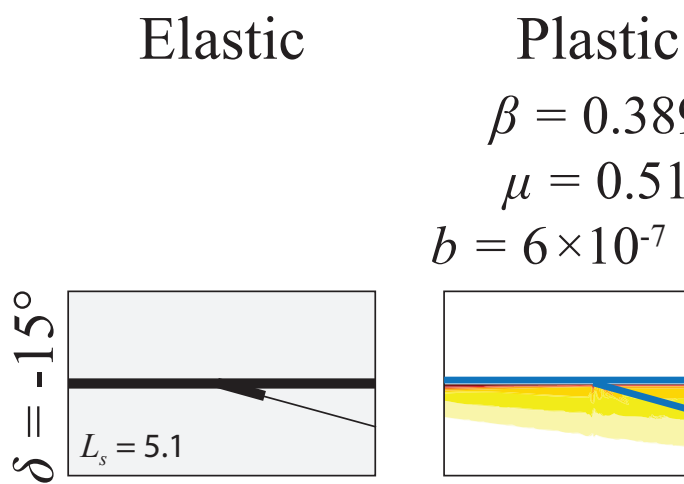

$$
\beta=0.389
$$$$
\mu=0.51
$$$$
b=6 \times 10^{-7}\left|\sigma_{y y}^{o}\right|
$$
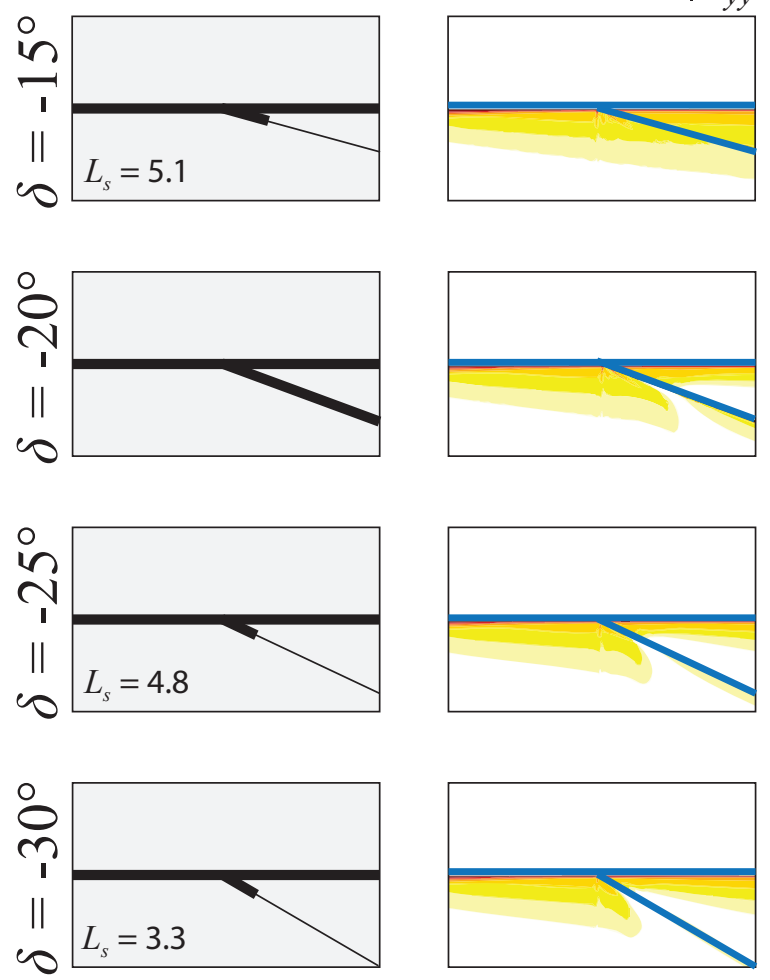

$\gamma_{e q}^{p l} \quad 2 \tau^{p} / G$

\section{\begin{tabular}{|l|l|l|l|l|l|l|}
\hline & & & & & & \\
\hline
\end{tabular}}

Figure 11: Influence of off-fault plastic deformation on branch activation. (a) Compressional side branch. (b) Extensional side branch. 

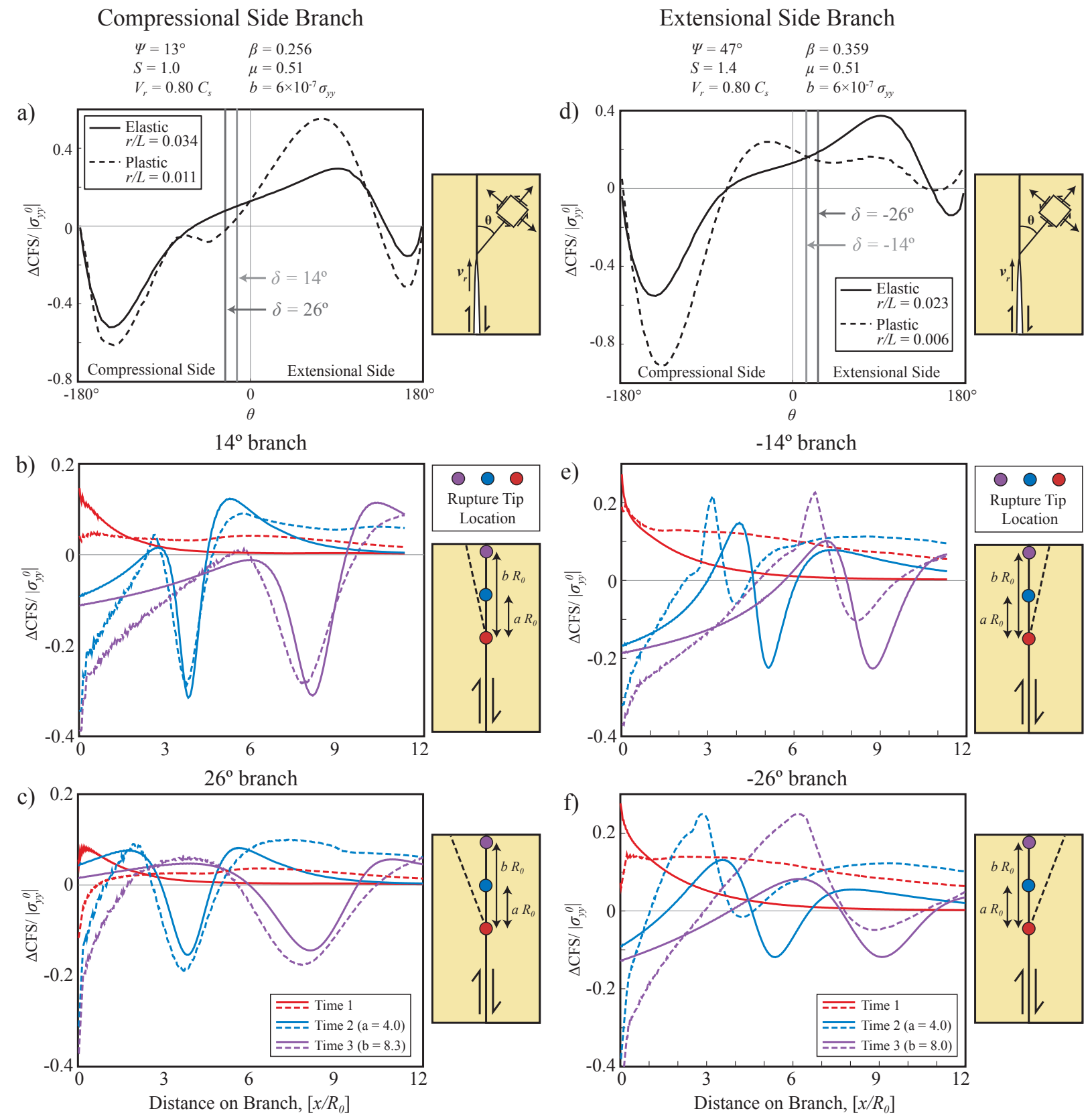

Figure 12: Change in CFS due to rupture propagation for elastic and elastic-plastic materials ( $\left.\Delta \mathrm{CFS}=\Delta \tau-0.6 \Delta \sigma_{n}\right)$. (a) - (c) Compressional side branch. (d) - (f) Extensional side branch. (a) \& (d) Stress distribution on all planes radiating from the rupture tip, at a distance $r / L$ from the tip. (b), (c), (e) and (f) Change in CFS on a fictional branch due to rupture propagating past the junction on the main fault. 


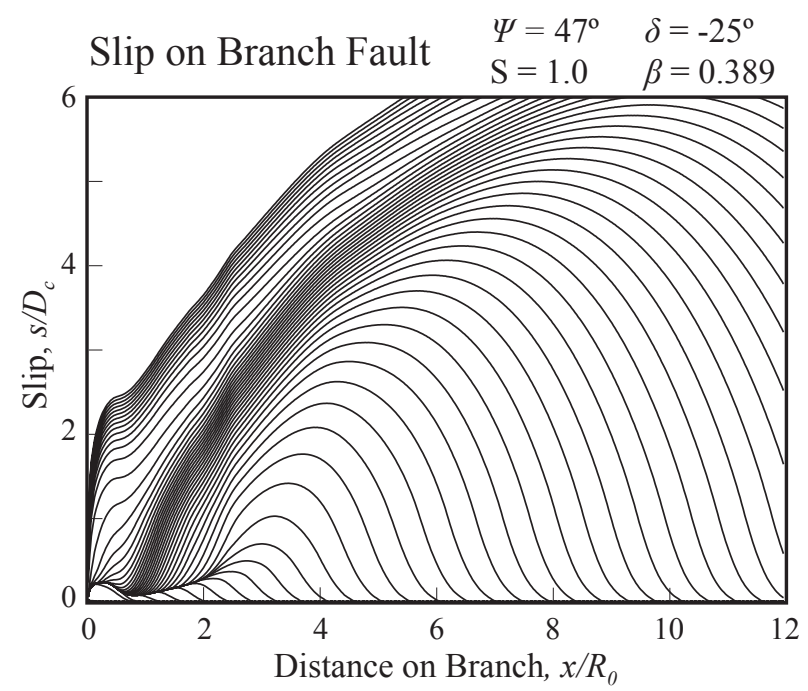

Figure 13: Slip distribution on the branch fault shown in figure $11 b, \delta=-25^{\circ}$. Lines plotted at equal time increments. Slip travels as a small pulse along the branch until a distance of 3.0, at which point full dynamic weakening occurs and the rupture goes bilateral to complete the rupture of the branch. 


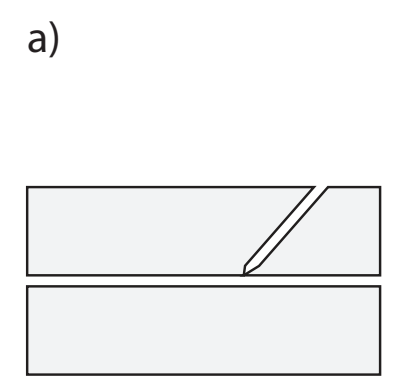

continuous through the main fault

b)

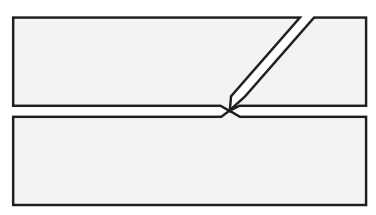

continuous through neither fault

c)

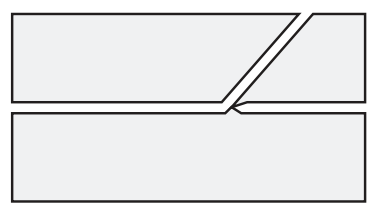

continuous through the branch fault
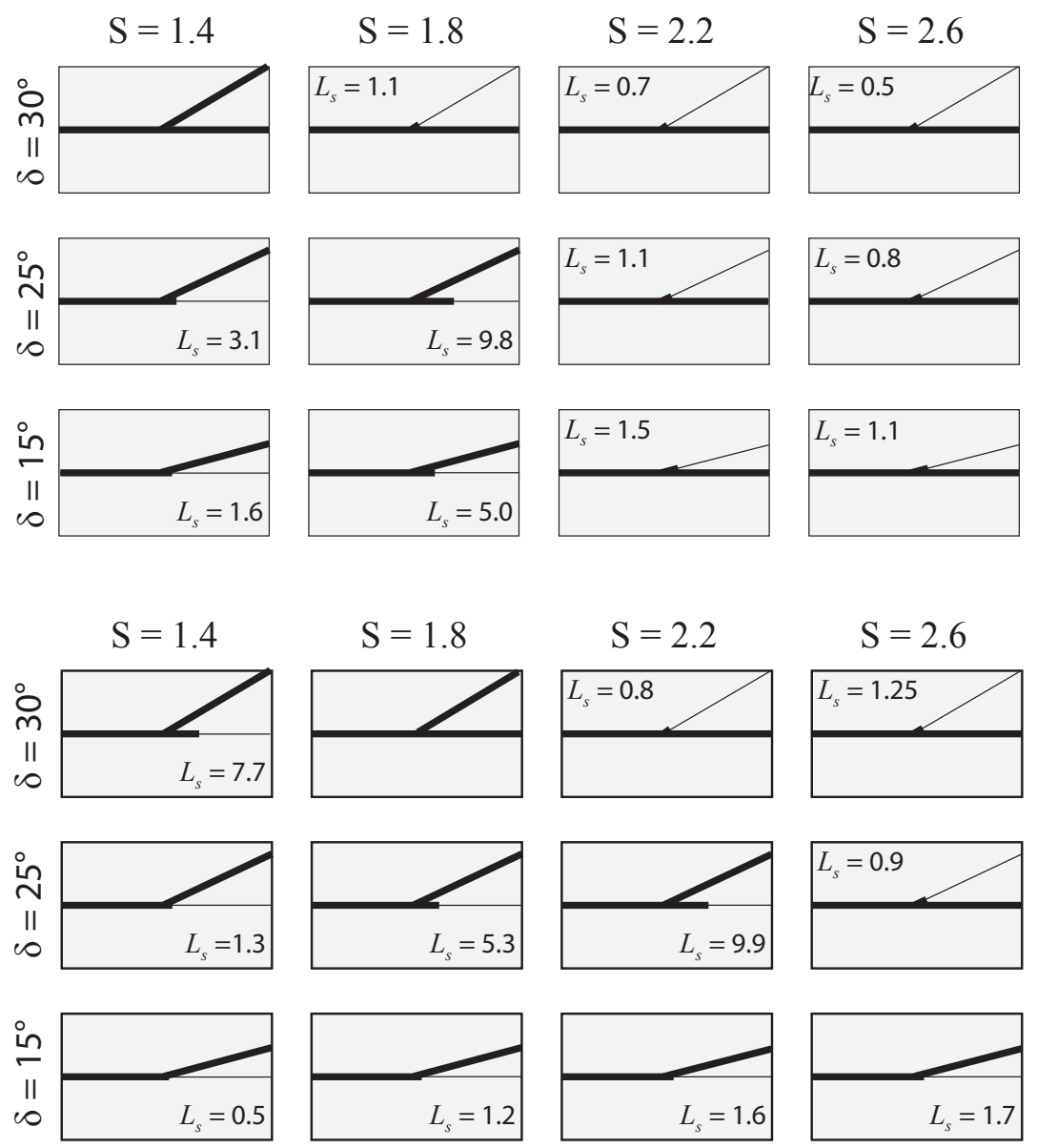

$\mathrm{S}=1.4$
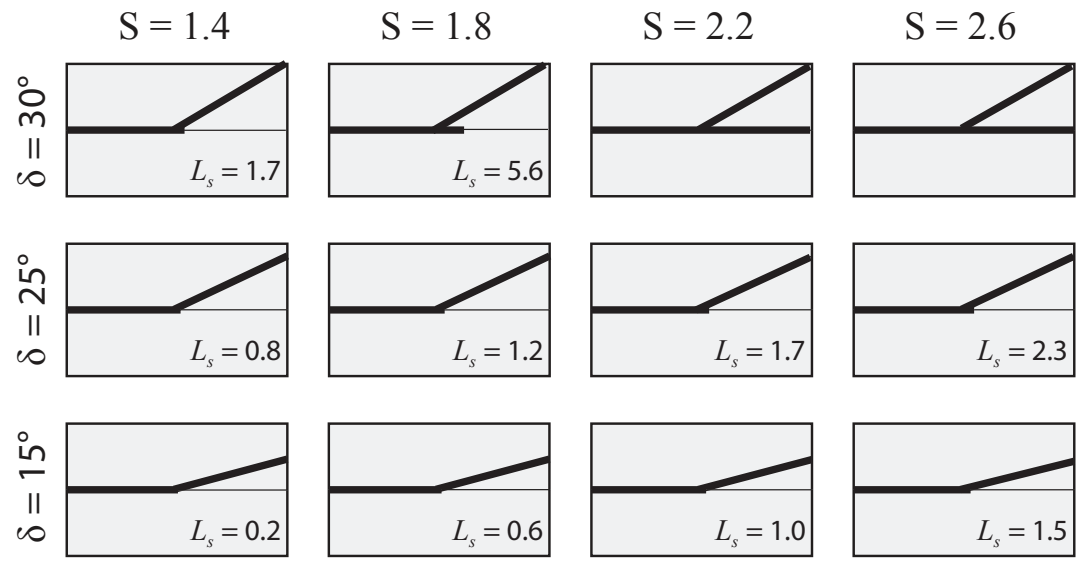

Figure 14: Effect of the definition at the branching junction on the rupture path selection. The rupture prefers to propagate on the continuous surface. All cases shown for $\Psi=13^{\circ}$ and $V_{r}=0.86 C_{s}$. As the $S$ ratio changes, so does the rupture path selection. $L_{s}$ indicates the distance of terminated rupture propagation, normalized by $R_{0}$. 


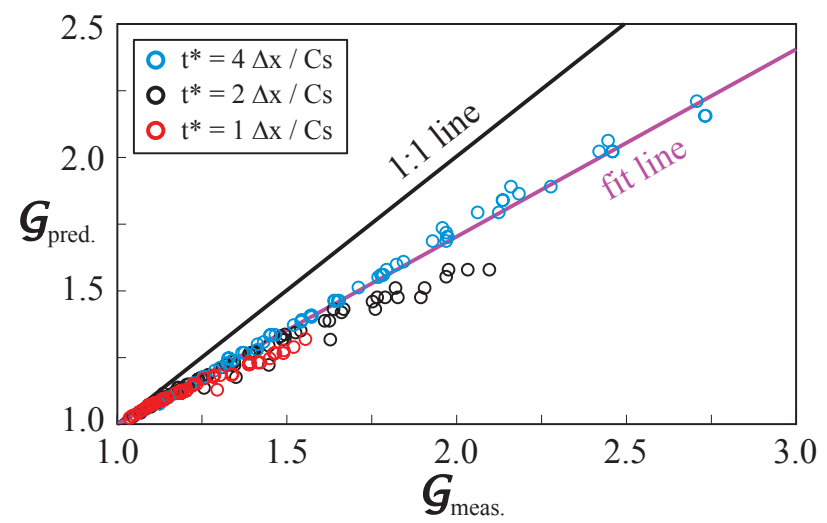

Figure 15: Predicted value for $\mathcal{G}$, from equations eq.(A13)-eq. (A16), shown against value measured for $\mathcal{G}$ from model runs. $\mathcal{G}$ is consistently under-predicted, but the use of a modified value for $T^{r}$ (see fit line) can predict the value. 